\title{
Metal-Organic Frameworks in Green Analytical Chemistry
}

\author{
Priscilla Rocío-Bautista $^{1,2,+}+\mathbb{C}$, Iván Taima-Mancera ${ }^{1,2,+}{ }^{,}$, Jorge Pasán ${ }^{2}(\mathbb{C})$ and Verónica Pino ${ }^{1,3, *(1)}$ \\ 1 Department of Chemistry, Analytical Division, University of La Laguna, 38206 Tenerife, Spain \\ 2 X-ray and Molecular Materials Lab (MATMOL), Physics Department, University of La Laguna, \\ 38206 Tenerife, Spain \\ 3 University Institute of Tropical Diseases and Public Health, University of La Laguna (ULL), La Laguna, \\ 38206 Tenerife, Spain \\ * Correspondence: veropino@ull.edu.es; Tel.: +34-922-318-990 \\ + These authors contribute equally to this work.
}

Received: 31 May 2019; Accepted: 21 June 2019; Published: 27 June 2019

check for updates

\begin{abstract}
Metal-organic frameworks (MOFs) are porous hybrid materials composed of metal ions and organic linkers, characterized by their crystallinity and by the highest known surface areas. MOFs structures present accessible cages, tunnels and modifiable pores, together with adequate mechanical and thermal stability. Their outstanding properties have led to their recognition as revolutionary materials in recent years. Analytical chemistry has also benefited from the potential of MOF applications. MOFs succeed as sorbent materials in extraction and microextraction procedures, as sensors, and as stationary or pseudo-stationary phases in chromatographic systems. To date, around 100 different MOFs form part of those analytical applications. This review intends to give an overview on the use of MOFs in analytical chemistry in recent years (2017-2019) within the framework of green analytical chemistry requirements, with a particular emphasis on possible toxicity issues of neat MOFs and trends to ensure green approaches in their preparation.
\end{abstract}

Keywords: metal-organic frameworks; analytical chemistry; sorbent materials; stationary phases; sensors; sample preparation; green considerations

\section{Introduction}

Metal-organic frameworks (MOFs) belong to a subclass of 3D coordination polymers, formed by metallic clusters and organic ligands through coordination bonds [1-3]. These hybrid materials are characterized by their crystallinity, ultra-low densities, permanent porosity, the presence of accessible cages, tunnels and modifiable pores, and exhibit the highest known surface areas [4,5]. Furthermore, they present adequate mechanical and thermal stability. The proper selection of certain metallic centers and specific ligands, together with a rational control of the synthetic approach, serve to design crystals with a controlled structure and topology, with the resulting MOF even being able to undergo post-modifications. This tuneability has resulted in the characterization of more than $80,000 \mathrm{MOFs}$ [6]. Figure 1 schematically presents well-known MOFs structures to highlight their impressive versatility.

Given this group of interesting properties, it is not surprising that these tailorable materials have been incorporated into an impressive number of applications within different scientific fields: as photovoltaic materials [7], in gas purification and separation [8,9], and gas storage systems [9,10], in catalysis [11], in biomedicine [12], etc. Analytical chemistry is not an exception; around a hundred MOFs form part of these studies [13]. MOFs are mainly involved in analytical chemistry as sorbents in analytical sample preparation methods [14-19], as stationary phases in analytical separation techniques [13,17,18,20,21], and as sensors in spectroscopy and/or electroanalytical methods [22-24]. 


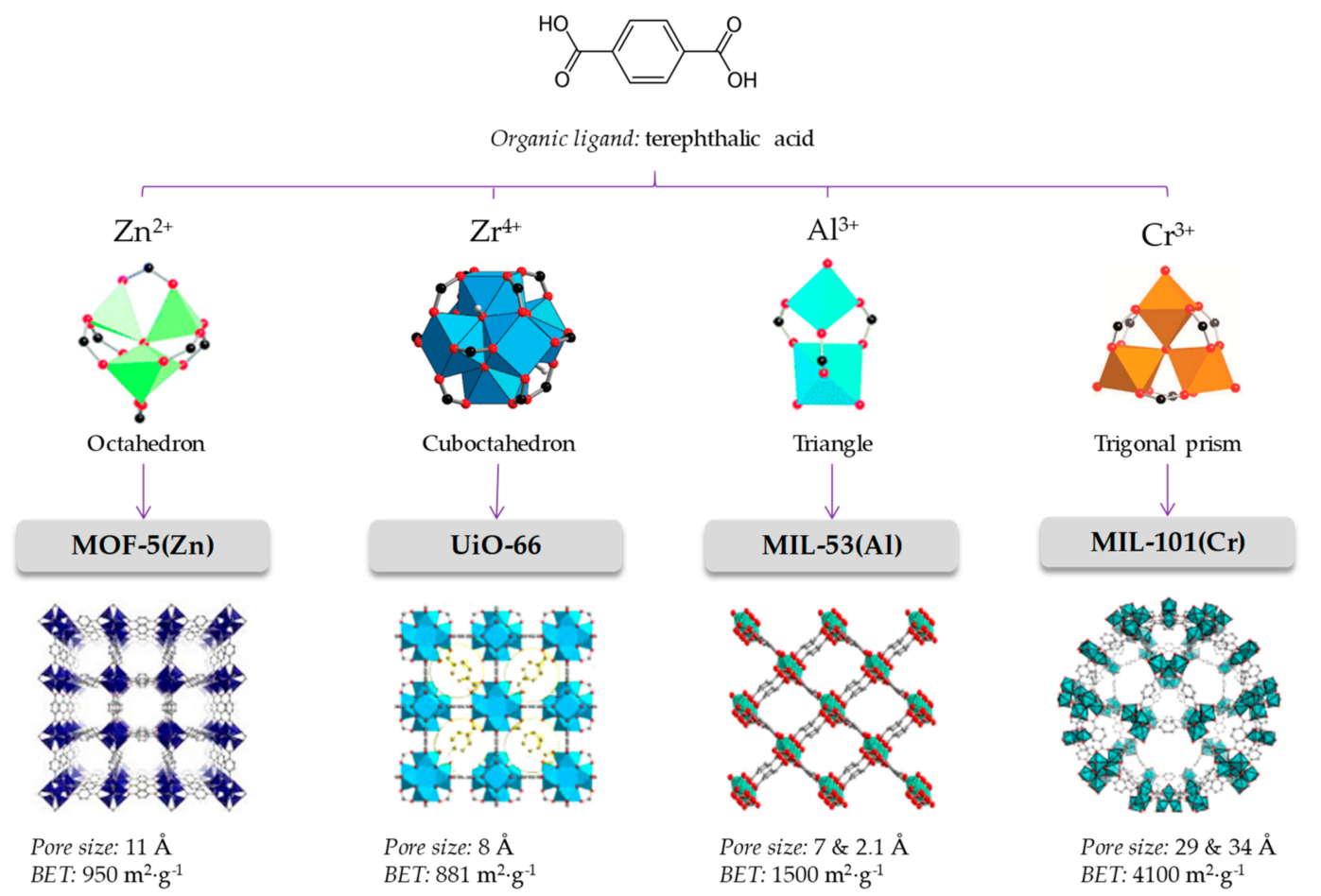

Figure 1. The node-and-connector approach to prepare MOFs. The adequate selection of the organic linker (linear in the case of terephthalic acid) and connection geometry of the metal cluster lead to the desired topology. Each framework topology has its characteristic pore size and available surface.

Current goals in analytical chemistry imply fulfilling as much as possible the requirements of green analytical chemistry (GAC) [25-27]. This way, the quality of an analytical method cannot link exclusively to its common quality features, e.g., accuracy, precision, detection limits, but it must also compile an estimation of method risks for the operator and for the environment [28,29]. GAC enforces the development of methods that ensure the elimination (or minimization of the consumption) of highly toxic chemical reagents (particularly toxic organic solvents), the minimization of gases emissions, the minimization of effluents and solid wastes discharges, the reduction of analysis times and number of analytical steps, and the incorporation of automation to the procedures [30-32]. When direct analysis is not possible due to sample complexity or difficulty in the analytical determination, miniaturization of the analytical method is the most common approach to follow GAC trends $[25,32,33]$. In any case, the use of novel solvents and/or sorbents, with environment-friendly characteristics is another important trend associated with the GAC approach [34,35].

Considering the rapid expansion of the analytical chemistry field of MOFs, it is important to consider that these and future analytical MOF-based methods must be incorporated into GAC. From this perspective, environment-friendly methods based on MOFs should include (partially or all) the following aspects: green design and synthesis of MOFs, evaluation of toxicity issues of MOFs, and incorporation of MOFs in GAC methods, mainly in miniaturized procedures. This review article will place particular emphasis upon trends which ensure the use of green approaches in the preparation of neat MOFs and/or in their incorporation into less-harmful analytical chemistry methods, limiting the overview to articles published in the years 2017-2019. It is also important to highlight that the attention will center upon bare MOF crystals rather than MOFs-composites. 


\section{Green Considerations during MOFs Preparation}

\subsection{MOFs Design}

The design of the MOF material constitutes a critical stage, primarily because the proper choice of metal centers and ligand connectivity is responsible of generating a particular crystal structure (a framework topology) with specific characteristics of pore size and window [36,37]. Metal centers act as nodes of the framework, and are characterized by the number and direction of its connecting positions. In Figure 1, for example, the $\mathrm{Zn}_{4} \mathrm{O}$ of MOF-5 is an octahedral node with six available connections located at the vertices of an octahedron. Ligands are characterized by their connectivity, or in other words, by how many metal centers can be linked. Thus, for example, in Figure 1, the terephthalic acid is a linear rod-like connector. The adequate combination of linker and nodes produces a particular topology; in the case of octahedral nodes with linear linkers, a primitive cubic framework is produced. When an application is pursued for a MOF, that application's requirements have to be taken into consideration. Therefore, a sorbent is required for an analytical sample preparation method, the desired MOF should be able to adsorb and release the analytes, while being stable in different solvents. If the MOF is going to be a chromatographic stationary phase, other considerations such as adequate permeability and high mechanical and/or thermal stability are additional aspects of interest.

This section will only focus on those aspects concerning the MOFs design that directly relate with the GAC considerations (assuming the obvious pursue of other important characteristics from an analytical chemistry performance). The main aspects to be considered when fabricating greener and sustainable metal-organic framework materials include: (i) Proper selection of the metal and metal source (reducing toxic metals and byproducts); and (ii) Adequate selection of the organic ligand (to ensure safer synthetic procedures and sustainable MOFs). The requirements in the MOF design and synthesis to comply for GAC issues and analytical application needs are summarized in Figure 2.

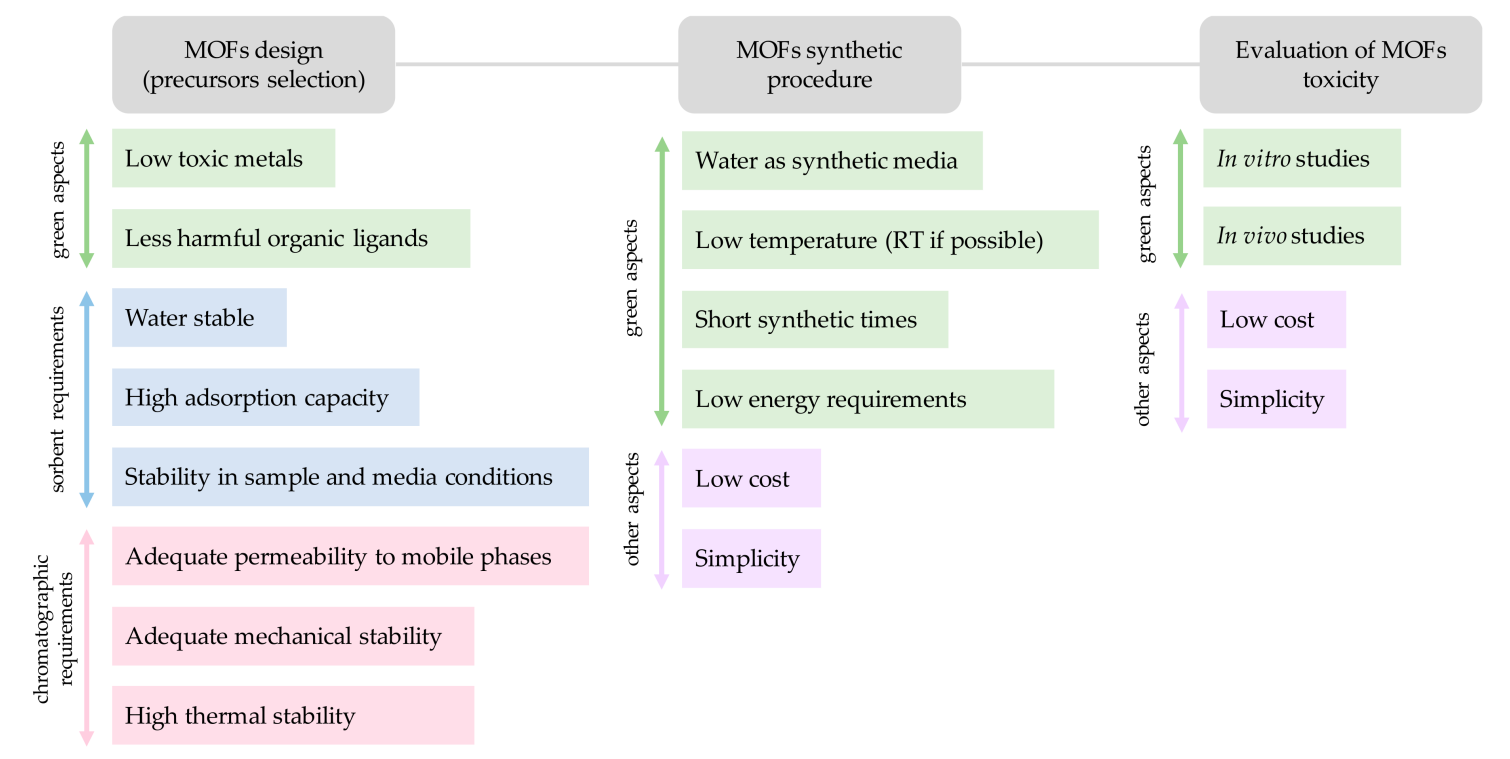

Figure 2. Considerations when preparing a MOF for analytical chemistry applications.

MOFs are chemical and physical stable materials, but in the event of degradation or decomposition, there are concerns due to the quantities of metal released to the environment. Thus, low-toxicity metal ions are preferable in the synthesis of MOFs, such alkaline earth metals ( $\mathrm{Mg}$ or $\mathrm{Ca}$ ), or $\mathrm{Mn}, \mathrm{Fe}, \mathrm{Al}$, $\mathrm{Ti}$ and $\mathrm{Zr}$ [38,39]. Another consideration when selecting metals is the handling of their salts when preparing MOFs. Thus, metal nitrates and perchlorates have risks of explosion due to oxidation upon handling, while chloride may induce corrosion. Metal oxides and hydroxides only liberate water as by-products, and are therefore ideal inorganic reactants; however, these salts often exhibit insufficient 
solubility/reactivity [40]. Another strategy to avoid problems with the anions of the metal salts is the use of zero-valence metal precursors and recently, the syntheses of MIL-53(Al), HKUST-1 or ZIF-7 have been reported to undergo from elementary metal precursors [41].

In any case, this general statement about ideal green metals for MOF does not mean that one cannot select other metals to prepare green MOFs or MOFs for green applications. For example, MOFs-containing rare earth metals are typically included in sensing [42], because the balance between the toxicity issues of the metal, the low amount involved in the sensor, and the benefits of the resulting material, clearly shift towards the selection of those metal ions. Finally, and even more importantly, the costs associated to the metal selection are of great significance when an industry-based application is concerned.

Regarding the organic linkers, some of the carboxylic acids are readily available in low cost, such as fumaric, succinic or terephthalic. However, tailored linkers used to reach the enormous surface areas reported for $\mathrm{MOF}$ materials in research laboratories often require multistep synthetic reactions with intermediates and byproducts. To comply with GAC requirements, the number of steps has to be as low as possible, while ensuring the low-toxicity of intermediates and byproducts. In general, it should be an equilibrium between low-toxicity and adsorption capacity of the MOF when tailoring a specific ligand for analytical applications. Figure 3 includes a group of representative organic ligands. In any case, it is advisable the use of simple and even biodegradable ligands in MOF design such as peptides, carbohydrates, amino acids, and cyclodextrins.

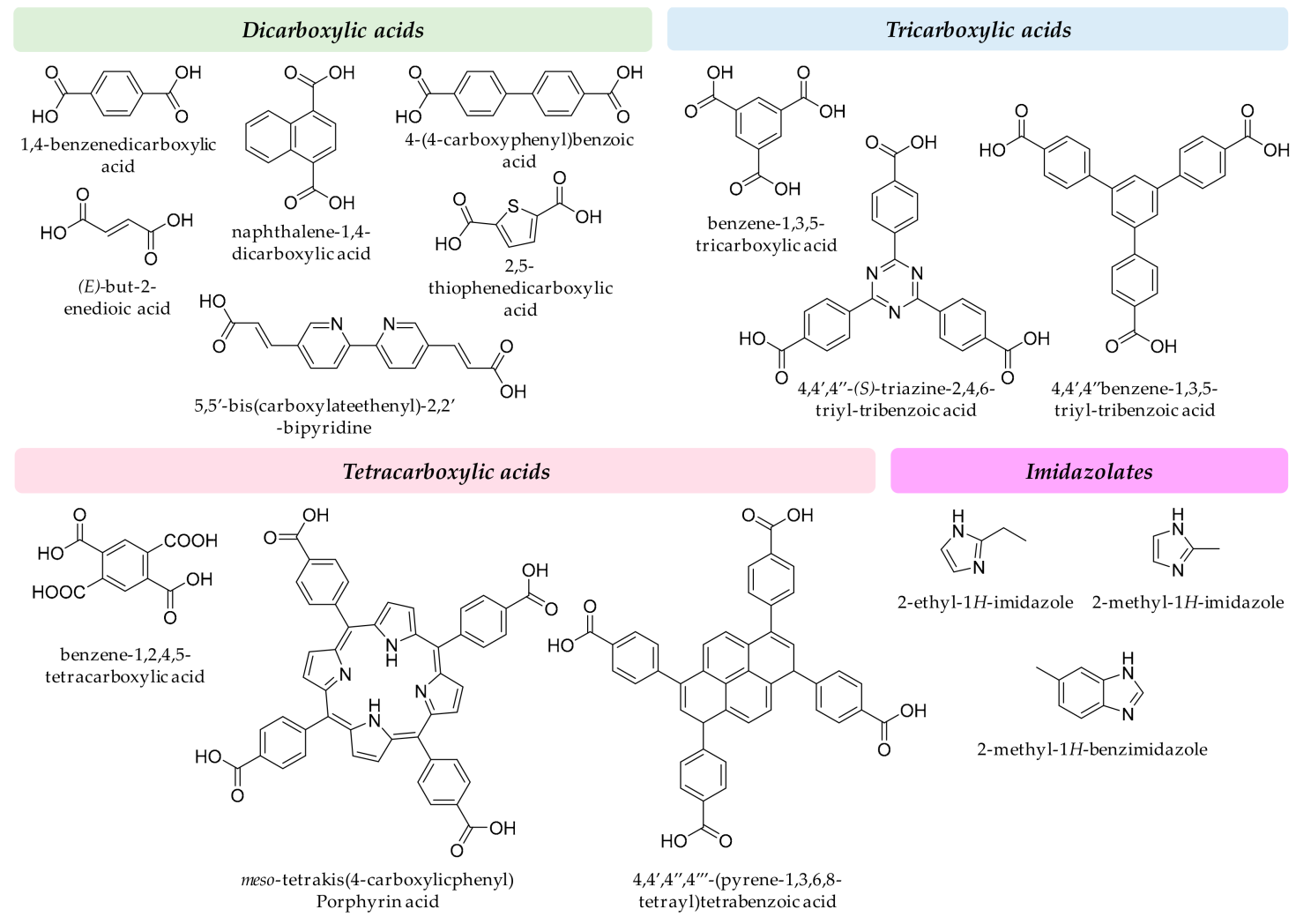

Figure 3. Representative examples of organic ligands used in MOF synthesis.

\subsection{MOFs Synthesis}

If there is a proper design of the MOF, thus implying adequate selection of precursors, the main parameters exerting an influence in the "green" synthesis of a MOF are: (i) type and duration of the input energy for the coordination bond formation, and (ii) solvent nature and volume [43]. 
Metal-organic frameworks can be synthesized following different crystallization procedures, although the most common strategy is the solvothermal method. In the solvothermal approach, the metal salt and the organic linker are mixed in a solvent (usually with a high boiling temperature). Then, this solution is introduced in an autoclave, followed by heating at an adequate temperature (between 80 and $300{ }^{\circ} \mathrm{C}$ ), during a certain time (in most cases, less than $96 \mathrm{~h}$ ). Other synthetic approaches (classified as a function of the energy input) include: microwave-assisted, electrochemical, mechanochemical, and sonochemical [44-47].

Recent advances in crystallization methods for MOF materials focus on environment-friendly alternatives to the classic solvothermal method [48]. It is worth noting that most of the reactions originally carried out by the solvothermal approach can also be performed in good yields through different, more environment-friendly, and also low-cost methods [46]. Microwave heating or ultrasound irradiation are simple, inexpensive and scalable routes towards the fabrication of MOFs. The reaction times using these techniques can be shortened, resulting in high-purity phases and high yields [49]. Mechanochemical synthesis has also appeared as a promising strategy to scale up the fabrication of MOFs. It is simple, and rapid, and although it may require some solvent addition (for example in liquid-assisted grinding, LAG), they are always in small amounts [47].

A simple change in the solvothermal reaction to make the MOF synthesis more sustainable implies the use of water instead of organic solvents. It must be noted that the most commonly used solvent, dimethylformamide, is a teratogen that readily decomposes at solvothermal temperatures into corrosive products. When using water, the main concern is the solubility of the reactants, but the use of the carboxylic acids in the form of sodium salts can help, while also precluding the formation of corrosive byproducts like $\mathrm{HNO}_{3}$ or $\mathrm{HCl}$ (coming from the metal salt and the deprotonation of the acid) [50]. Other green solvents can be used to better comply with the GAC requirements, among them, ionic liquids or urea derivatives [51]. In recent years, an increasing number of hydrothermal preparations of MOFs have been accomplished at atmospheric pressure with promising results in term of yield and simplicity [52-55].

As a final consideration regarding greener synthesis of MOFs, it would be advisable to use chemometric tools during the optimization of the synthesis, such as an experimental design [56,57], rather than a one-factor-at-a-time optimization. This type of procedure would make it possible to minimize time and quantities of reagents when preparing an adequate MOF, and thus, would be in consonance with green chemistry.

\subsection{Evaluation of MOFs Toxicity}

The few studies dealing with the toxicity evaluation of MOFs are mostly related to in vitro cytotoxicity studies [58,59], which were usually performed to evaluate the performance of those MOFs for use as drug nano-carriers [60]. Moreover, the majority of these studies focuses on MOFs included in composites forming such carriers. In fact, most studies do not try to evaluate the MOF cytotoxicity itself, but, in general, to compare with the composite in which the MOF is included.

In the reported in vitro cytotoxicity studies, different cell lines or macrophages incubate in presence of MOFs suspensions for a certain amount of time. Afterwards, because of the appearance in the culture media of a compound coming from the cell lysis, a colorimetric detection is commonly used. The released compound is then able to react with the specific reagent of the cytotoxicity test. Among in vitro cytotoxicity tests, MTT [58,61-65], the alamarBlue ${ }^{\circledR}$ cell viability assay [54,66], and CCK-8 [67], are the most widely used with MOFs. Table 1 includes a list of cytotoxicity tests performed with neat MOFs so far, resulting in adequate cytotoxicity values [54,58-61,63,65,67-70]. These results support the fact that it results safe the use of relatively high concentrations of these MOFs aqueous suspensions. As a note, all these results must be taken only as qualitative data with MOFs, because conventional cytotoxicity studies are designed for soluble compounds (and only water suspensions can be prepared with MOFs). It is also important to mention that cell lines may also play an important role in the toxicity assays performed since for example macrophages are more robust cells than HeLa ones. An alternative 
approach to evaluate these MOFs suspensions should be proposed since the assays described so far are likely to be distant from what it is actually occurring in real conditions.

Table 1. Representative examples of cytotoxicity studies of MOFs.

\begin{tabular}{|c|c|c|c|c|}
\hline MOF (metallic ion \& ligand) & $\begin{array}{l}\text { Cytotoxicity } \\
\text { Test Type }\end{array}$ & Cell Line Type & $\begin{array}{l}\text { Toxicity Value } \\
\quad\left(\mathrm{mg} \cdot \mathrm{L}^{-1}\right)\end{array}$ & Ref. \\
\hline $\mathrm{UiO}-66\left(\mathrm{Zr}^{4+} \& \mathrm{H}_{2} \mathrm{bdc}^{\mathbf{1}}\right)$ & $\mathrm{MTT}^{2}$ & MCF-7 & $\begin{array}{l}>80 \mathrm{mg} \cdot \mathrm{L}^{-1} \\
1100 \& 700\end{array}$ & {$[61]$} \\
\hline $\begin{array}{l}\text { MIL-100(Fe) }\left(\mathrm{Fe}^{3+} \& \mathrm{H}_{3} \mathrm{btc}^{3}\right) \\
\text { UiO-66 }\left(\mathrm{Zr}^{4+} \& \mathrm{H}_{2} \mathrm{bdc}^{1}\right) \\
\text { ZIF-8 }\left(\mathrm{Zn}^{2+} \& 2-\mathrm{MIm}^{4}\right) \\
\text { among many others }\end{array}$ & MTT $^{2}$ & $\begin{array}{l}\text { HeLa \& J774.2 } \\
\text { HeLa \& J774.2 } \\
\text { HeLa \& J774.2 }\end{array}$ & $\begin{array}{c}\mathrm{mg} \cdot \mathrm{L}^{-1} \\
400 \& 60 \\
\mathrm{mg} \cdot \mathrm{L}^{-1} \\
100 \& 25 \\
\mathrm{mg} \cdot \mathrm{L}^{-1}\end{array}$ & [58] \\
\hline $\begin{array}{l}{\left[\mathrm{Zn}_{6}(\mathrm{~L})_{3}(\mathrm{DMA})_{4}\right] \cdot 5 \mathrm{DMA}} \\
\left(\mathrm{Zn}^{2+} \& \mathrm{H}_{4} \mathrm{~L}^{5}\right)\end{array}$ & $\mathrm{MTT}^{2}$ & Hela \& HEK293 & $5000 \mathrm{mg} \cdot \mathrm{L}^{-1}$ & {$[63]$} \\
\hline $\operatorname{MIL}-101(\mathrm{Cr})\left(\mathrm{Cr}^{3+} \& \mathrm{H}_{2} \mathrm{bdc}^{\mathbf{1}}\right)$ & MTT $^{2}$ & $\begin{array}{l}\text { Hep-2 } \\
\text { MCF-7 }\end{array}$ & $\begin{array}{l}744 \mathrm{mg} \cdot \mathrm{L}^{-1} \\
200 \mathrm{mg} \cdot \mathrm{L}^{-1}\end{array}$ & {$[64]$} \\
\hline $\mathrm{ZIF-8}\left(\mathrm{Zn}^{2+} \& 2-\mathrm{MIm}^{4}\right)$ & MTT $^{2}$ & $\begin{array}{l}\text { MDA-MB-231 } \\
\text { MDA-MB-468 }\end{array}$ & $\begin{array}{l}400 \mathrm{mg} \cdot \mathrm{L}^{-1} \\
400 \mathrm{mg} \cdot \mathrm{L}^{-1}\end{array}$ & [65] \\
\hline $\begin{array}{l}\text { NanoMOF based on } \mathrm{Fe} \\
\left(\mathrm{Fe}^{3+} \& \mathrm{TCPP}^{6}\right)\end{array}$ & MTT $^{2}$ & $4 \mathrm{~T} 1$ & $>200 \mathrm{mg} \cdot \mathrm{L}^{-1}$ & [68] \\
\hline MIL-100(Fe) $\left(\mathrm{Fe}^{3+} \& \mathrm{H}_{3} \mathrm{btc}^{3}\right)$ & MTT $^{2}$ & \multirow{2}{*}{$\begin{array}{l}\text { HL-7702 \& HepG2 } \\
\text { J774.A1 }\end{array}$} & $80 \mathrm{mg} \cdot \mathrm{L}^{-1}$ & [59] \\
\hline $\begin{array}{l}\text { MIL-88A }\left(\mathrm{Fe}^{3+} \& \text { fumaric acid }\right) \\
{\left[\mathrm{Zn}\left(\mathrm{H}_{3} \mathrm{btc}^{3}\right)\left(\mathrm{HME}^{7}\right)\right]}\end{array}$ & MTT $^{2}$ & & \multirow[b]{2}{*}{$100 \mathrm{mg} \cdot \mathrm{L}^{-1}$} & {$[60]$} \\
\hline $\begin{array}{l}\left(\mathrm{DMAc}^{8}\right)\left(\mathrm{H}_{2} \mathrm{O}\right) \\
\left(\mathrm{Zn}^{2+} \& \mathrm{H}_{3} \mathrm{btc}^{3} \& \mathrm{HME}^{7}\right)\end{array}$ & MTT $^{2}$ & SCC-251 \& HSC-4 & & [69] \\
\hline $\mathrm{UiO}-64\left(\mathrm{Zr}^{4+} \&\right.$ fumaric acid $)$ & MTT $^{2}$ & J774 \& PBLs & \multirow{3}{*}{$\begin{array}{c}200 \mathrm{mg} \cdot \mathrm{L}^{-1} \\
>2000 \mathrm{mg} \cdot \mathrm{L}^{-1} \\
>80 \mathrm{mg} \cdot \mathrm{L}^{-1} \\
20 \mathrm{mg} \cdot \mathrm{L}^{-1}\end{array}$} & {$[70]$} \\
\hline CIM-80 ( $\mathrm{Al}^{3+} \&$ mesaconic acid $)$ & alamarBlue ${ }^{\circledR 9}$ & $\mathrm{~J} 774.1$ & & [54] \\
\hline $\begin{array}{l}\text { ZIF-67 }\left(\mathrm{Co}^{2+} \& 2-\mathrm{MIm}^{4}\right) \\
\text { ZIF-8 }\left(\mathrm{Zn}^{2+} \& 2-\mathrm{MIm}^{4}\right)\end{array}$ & CCK-8 ${ }^{10}$ & $293 \mathrm{~T}$ & & [67] \\
\hline \multicolumn{2}{|c|}{$\begin{array}{l}1 \text { terephthalic acid (benzene-1,4-dicarboxylic acid) } \\
23 \text {-(4,5-dimethylthiazol-2-yl)-2,5-diphenyltetrazolium bromide dye } \\
3 \text { trimesic acid (benzene-1,3,5-tricarboxylic acid) } \\
4 \text { 2-methylimidazole } \\
5 \text { [1,1':3',1"-terphenyl]-3,3",5,5"-tetracarboxylic acid } \\
6 \text { tetrakis(4-carboxyphenyl)porphyrin }\end{array}$} & $\begin{array}{l}{ }^{7} \text { proton } \\
8 \mathrm{~N}, N \text {-di } \\
{ }^{9} \text { from re } \\
\text { (fluoresc } \\
{ }^{10} \text { cell-co } \\
\text { (colorles }\end{array}$ & $\begin{array}{l}\text { mine } \\
\text { etamide } \\
\text { olue) to resorufin } \\
\text { ink) } \\
\text { t assay, from WS } \\
-8 \text { formazan dye }\end{array}$ & ye \\
\hline
\end{tabular}

Tamames-Tabar et al. studied thoroughly the in vitro cytotoxicity of up to $14 \mathrm{MOFs}$. Among other interesting results, authors observed that there were no differences in the cytotoxicity of polymorphs, thus indicating a poor effect of the MOF topology into the resulting cytotoxicity [54]. The same authors also showed that, for the same type of linker, Zn-based MOFs were more cytotoxic than Zr-based MOFs, being the less cytotoxic those MOFs based on Fe [54]. From the group of 14 MOFs tested, in vivo cell penetration studies were carried out only with MIL-100(Fe) for being the best candidate as drug nano-carrier. The studies indicated an immediate cell internalization in J774 mouse macrophages, faster than in epithelial HeLa cell lines [54].

In the last two years, an increasing number of studies have been undertaken on the effects of MOFs on living cells from safety aspects while also taking into account therapeutic considerations [71]. Thus, Shen et al. demonstrated that the MOF MIL-101(Fe) functionalized with amino groups was able to induce cyto-protective autophagy in mouse embryonic fibroblast cells instead of cytotoxicity [71].

\section{Analytical Methods Incorporating MOFs}

Nowadays, the main uses of neat MOFs in analytical chemistry range from analytical sample preparation (as novel sorbents) to chromatography (as novel stationary or pseudo-stationary phases) and sensing (as novel materials in the sensors) [13]. In the majority of these applications, the GAC requirements [72] cover partially by incorporating the MOFs in microextraction approaches within sample preparation, by requiring quite low amounts of MOFs when preparing the chromatographic phases, or by incorporating minimum amounts of MOFs in the sensors. In few applications, it is also possible to find many GAC aspects fulfilled, that is: green MOF designed, MOF as prepared following a green synthesis and demonstrating low cytotoxicity, plus incorporation in a miniaturized method [54]. 


\subsection{MOFs in Analytical Sample Preparation}

Improvements in analytical extraction methods from a GAC perspective include the reduction of analysis times by using, for example, ultrasounds or microwaves to accelerate the extraction step, particularly when dealing with solid samples [73]. Regarding miniaturization, GAC approaches include the generic liquid-phase microextraction (when the extraction solvent amount rarely exceeds $0.5 \mathrm{~mL}$ ) $[74,75]$ and solid-based miniaturized extraction (when the extraction sorbent amount is below $0.5 \mathrm{~g}$ ) [76-78] methods. Among sorbent-based miniaturized approaches, it is possible to distinguish many sub-modes. Thus, micro-solid-phase extraction ( $\mu$-SPE) is quite similar to conventional solid-phase extraction, but requiring lower sorbent amounts in devices such as micro-columns, syringe tips or bodies, mini-disks, etc. Dispersive miniaturized solid-phase extraction ( $\mu$-dSPE) utilizes the sorbent material in direct contact with the sample, followed by further separation and desorption. The magnetic-based version (m- $\mu$-dSPE) needs a magnetic material as sorbent, thus avoiding further centrifugation and/or centrifugations steps during the method, because the sorbent separates easily from the sample with the aid of an external magnet. Solid-phase microextraction (SPME), in its more conventional version, utilizes thin fibers coated with a sorbent material, whereas stir-bar sorptive microextraction (SBSME) uses a stir bar coated with a sorbent material.

Table 2 shows representative examples of performance for different analytical sorbent-based microextraction techniques ( $\mu$-SPE, $\mu$-dSPE, $\mathrm{m}-\mu$-dSPE, SPME and SBSME) incorporating MOFs as novel sorbent materials [54,79-99].

If we take into consideration the idea of avoiding the use of heavy metals when designing MOFs, it is clear that many MOFs included in Table 2 present non-toxic metallic ionic centers [54,65], such as $\mathrm{Zn}^{2+}$ [93], $\mathrm{Zr}^{4+}$ [82] or $\mathrm{Al}^{3+}$ [54]. Regarding the synthetic solvents in MOFs preparations, most studies employ DMF $[82,90,94]$, avoiding in this way toxic chlorinated ones, such as dichloromethane or chloroform. Furthermore, the DMF volumes required are usually low (around $30 \mathrm{~mL}$ for $15 \mathrm{mg}$ of MOF). It is noticeable that several MOFs used in these microextraction methods include water as solvent in their preparation (hydrothermal synthesis) $[54,81]$. The temperatures needed during the preparation of these crystals are usually moderate, i.e., between 80 and $150{ }^{\circ} \mathrm{C}[94,96]$, and sometimes even room temperature [93]. Regarding the synthetic times required, they are usually less than $24 \mathrm{~h}$ [83,88], and even down to one hour or half an hour $[80,83]$. Recent publications have favored the utilization of so-called bio-MOFs [90], incorporating a bioorganic ligand in its structure (i.e., adenine).

In general, quite low amounts of MOF as sorbents are always needed $[54,82,84]$ in all microextraction procedures listed, from $2 \mathrm{mg}$ [80] to $60 \mathrm{mg}$ [89]. Regarding extraction times, there are reported values around $3 \mathrm{~min}$ or even less [85] for $\mu$-dSPE and some of its applications in the magnetic variant $[89,91]$.

If we focus on the analytical applications, microextraction techniques using MOFs commonly determine organic pollutants in environmental and food samples [86,88], from drugs in waters [90] to PAHs in foods [96]; and also trace metals in food and water samples [80,83]. 
Table 2. Representative examples of environment-friendly microextraction methods incorporating MOFs as novel sorbents in the period $2017-2019$.

\begin{tabular}{|c|c|c|c|c|c|c|}
\hline $\begin{array}{l}\text { MOF (metallic ion \& ligand) } \\
\text { Synthetic solvent }(\mathrm{mL}) / \mathrm{T}\left({ }^{\circ} \mathrm{C}\right) / \text { time }(\mathrm{h})\end{array}$ & $\begin{array}{l}\text { Microextraction format/Extraction } \\
\text { time (min) }\end{array}$ & MOF amount & Analytes (number)/Sample (amount) & $\operatorname{LOD}^{1}\left(\mathbf{n g} \cdot \mathrm{L}^{-1}\right) / \mathrm{RSD}^{2}(\%)$ & Analytical technique & Ref. \\
\hline \multicolumn{7}{|c|}{$\mu$-SPE } \\
\hline $\begin{array}{l}\mathrm{UiO}-66 \\
\left(\mathrm{Zr}^{++} \& \mathrm{H}_{2} \mathrm{bdc}^{3}\right) \\
\mathrm{DMF}^{4}(40) / 120 / 24\end{array}$ & $\begin{array}{l}\mathrm{PP}^{5} \text { cartridge (packing the MOF } \\
\text { suspension)/40 }\end{array}$ & $10 \mathrm{mg}$ & $\begin{array}{l}\text { hormones (4)/waters } \\
\quad(20 \mathrm{~mL})\end{array}$ & $2.0-10 /<6.5$ & $\mathrm{LC}^{6}-\mathrm{MS} / \mathrm{MS}^{7}$ & [79] \\
\hline $\begin{array}{l}\mathrm{PCN}-222 \\
\left(\mathrm{Zr}^{4+} \& \mathrm{H}_{2} \mathrm{TCPP}^{8}\right) \\
\mathrm{DMF}^{4}(40) / 100 / 1\end{array}$ & $\begin{array}{l}\text { pipette tip (packing the MOF } \\
\text { powder) } /<7\end{array}$ & $2 \mathrm{mg}$ & $\begin{array}{c}\mathrm{Hg}^{2+}(1) / \text { fish } \\
(100 \mathrm{~mL} \text { aqueous extract) }\end{array}$ & $20 / 8$ & CVAAS $^{9}$ & [80] \\
\hline \multicolumn{7}{|c|}{$\mu$-dSPE } \\
\hline $\begin{array}{l}\text { MIL-53(Al) } \\
\left(\mathrm{Al}^{3+} \& \mathrm{H}_{2} \mathrm{bdc}^{3}\right) \\
\text { water }(-) / 210 / 72\end{array}$ & MOF powder/30 & $8 \mathrm{mg}$ & hormones $(8) /$ water \& urine $(8 \mathrm{~mL})$ & $1.5-1000 /<7.8$ & $\mathrm{LC}^{6}-\mathrm{MS} / \mathrm{MS}^{7}$ & [81] \\
\hline 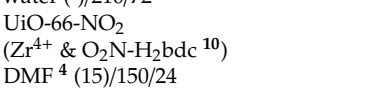 & MOF powder/3 & $20 \mathrm{mg}$ & $\begin{array}{l}\text { EDCs }{ }^{11}(9) / \text { waters } \\
\quad(20 \mathrm{~mL})\end{array}$ & $1.5-90 /<14$ & $\mathrm{LC}^{6}-\mathrm{DAD}^{12}$ & [82] \\
\hline $\begin{array}{l}\text { Al-Fu nano-flakes } \\
\left.\text { (A } \mathrm{A}^{3+} \& \text { fumaric acid }\right) \\
\text { water }(200) / 90 / 0.5\end{array}$ & MOF powder $/ 20 \mathrm{~s}$ & $30 \mathrm{mg}$ & $\begin{array}{c}\mathrm{Cu}^{2+}(1) / \text { waters \& food ( } 25 \mathrm{~mL} \text { sample or } \\
\text { aqueous extract) }\end{array}$ & $0.2 \mu \mathrm{g} \cdot \mathrm{L}^{-1} / 6.5$ & GFAAS ${ }^{13}$ & [83] \\
\hline $\begin{array}{l}\text { CIM- } 81 \\
\left(\mathrm{Zn}^{2+} \& \mathrm{Htz}^{14}+\mathrm{H}_{2} \mathrm{bdc}^{3}\right) \\
\text { DMA }^{15}(15) / 120 / 72\end{array}$ & MOF powder/1 & $10 \mathrm{mg}$ & $\begin{array}{l}\text { PCPs }{ }^{16}(9) / \text { waters } \\
\quad(10 \mathrm{~mL})\end{array}$ & $0.5-1.5 \mu \mathrm{g} \cdot \mathrm{L}^{-1} /<13$ & $\mathrm{LC}^{6}-\mathrm{UV}^{17}$ & [84] \\
\hline $\begin{array}{l}\text { CIM-80 } \\
\left(\mathrm{Al}^{3+} \& \text { mesaconic acid }\right) \\
\text { water }(15) / 150 / 3\end{array}$ & MOF powder/3 & $20 \mathrm{mg}$ & $\begin{array}{c}\text { PAHs }^{18}(15) \& \text { EDCs }^{11}(7) / \text { waters } \\
(10 \mathrm{~mL})\end{array}$ & $\begin{array}{c}0.75-9.3(\mathrm{PAHs}) \& 0.11-21 \\
\mu \mathrm{g} \cdot \mathrm{L}^{-1}(\mathrm{EDCs}) /<19\end{array}$ & $\begin{array}{l}\mathrm{LC}^{6}-\mathrm{UV}^{17} \\
\& \mathrm{LC}^{6}-\mathrm{FD}^{19}\end{array}$ & [54] \\
\hline 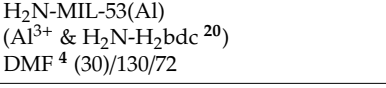 & MOF powder/10 s & $30 \mathrm{mg}$ & $\begin{array}{l}\text { phenols }(8) / \text { waters } \\
(10 \mathrm{~mL})\end{array}$ & $0.4-13.3 \mu \mathrm{g} \cdot \mathrm{L}^{-1} /<6.30$ & $\mathrm{LC}^{6}-\mathrm{PDA}^{21}$ & [85] \\
\hline \multicolumn{7}{|c|}{$m-\mu-d S P E$} \\
\hline $\begin{array}{l}\mathrm{Fe}_{3} \mathrm{O}_{4}- \\
\mathrm{CO}_{2} \mathrm{H}^{-} @ M I L-101-\mathrm{NH}_{2} \\
\left(\mathrm{Fe}^{3+} \& \mathrm{NH}_{2}-\mathrm{H}_{2} \mathrm{bdc}^{20}\right) \\
\mathrm{DMF}^{4}(15) / 110 / 24\end{array}$ & $\begin{array}{l}\text { heterogeneous composite } \\
\text { powder } / 20\end{array}$ & $20 \mathrm{mg}$ & fungicides (4)/waters (30) & $0.04-0.4 \mu \mathrm{g} \cdot \mathrm{L}^{-1} /<10.2$ & $\mathrm{LC}^{6}{ }^{6} \mathrm{UV}^{17}$ & [86] \\
\hline $\begin{array}{l}\mathrm{magG} 22 @ \mathrm{ZIF}-8 \\
\left(\mathrm{Zn}^{2+} \& 2-\mathrm{MIm}^{23}\right) \\
\text { methanol }(60) / \mathrm{RT}^{24} / 24\end{array}$ & $\begin{array}{l}\text { heterogeneous composite } \\
\text { powder/10 }\end{array}$ & $10 \mathrm{mg}$ & $\begin{array}{c}\text { PAEs }{ }^{25} \text { (9)/diluted human plasma }(1: 3, \\
\text { overall volume } \sim 9 \mathrm{~mL})\end{array}$ & $\begin{array}{c}3-10 /< \\
6.5\end{array}$ & $\mathrm{GC}^{26}-\mathrm{MS}^{7}$ & [87] \\
\hline $\begin{array}{l}\mathrm{Fe}_{3} \mathrm{O}_{4} / \mathrm{GO}^{27}-\mathrm{IRMOF}^{2}-3 \\
\left(\mathrm{Zn}^{2+} \& \mathrm{NH}_{2}-\mathrm{H}_{2} \mathrm{bdc} \mathrm{bc}^{20}\right) \\
\mathrm{DMF}^{4}(40) / \mathrm{RT}^{24} / 3\end{array}$ & $\begin{array}{l}\text { heterogeneous composite } \\
\text { powder } / 30\end{array}$ & $10 \mathrm{mg}$ & $\begin{array}{l}\text { fungicides (5)/lettuce } \\
\text { (10 mL aqueous extract) }\end{array}$ & $0.25-1.0 \mu \mathrm{g} \cdot \mathrm{L}^{-1} /<7.3$ & $\mathrm{LC}^{6}-\mathrm{MS} / \mathrm{MS}^{7}$ & [88] \\
\hline $\begin{array}{l}\mathrm{Fe}_{3} \mathrm{O}_{4} @ \mathrm{PDA}{ }^{28} @ \\
\mathrm{MIL}^{20}-101(\mathrm{Fe})\left(\mathrm{Fe}^{3+} \& \mathrm{H}_{2} \mathrm{bdc}^{3}\right) \\
\mathrm{DMF}^{4}(80) / 110 / 24\end{array}$ & heterogeneous composite powder $/ 3$ & $60 \mathrm{mg}$ & $\begin{array}{l}\text { SUHs }{ }^{29}(4) / \text { waters \& vegetables }(25 \mathrm{~mL} \\
\text { sample or aqueous extract) }\end{array}$ & $0.12-0.34 \mu \mathrm{g} \cdot \mathrm{L}^{-1} /<4.8$ & $\mathrm{LC}^{6}-\mathrm{PDA}^{21}$ & [89] \\
\hline $\begin{array}{l}\mathrm{Fe}_{3} \mathrm{O}_{4}-\mathrm{NH}_{2} / \text { bio-MOF-1 } \\
\left(\mathrm{Zn}^{2+} \& \text { adenine) }\right. \\
\mathrm{DMF}^{4}(67.5) / 130 / 24\end{array}$ & $\begin{array}{l}\text { heterogeneous composite } \\
\text { powder } / 40\end{array}$ & $15 \mathrm{mg}$ & $\mathrm{BZPS}^{30}(6) /$ urine \& waters $(40 \mathrm{~mL})$ & $0.71-2.49 /<8.8$ & $\mathrm{LC}^{6}-\mathrm{MS}^{7}$ & [90] \\
\hline $\begin{array}{l}\mathrm{Fe}_{3} \mathrm{O}_{4} @ \\
\text { TMU-10 } \\
\left(\mathrm{Co}^{2+} \& \mathrm{H}_{2} \mathrm{oba}^{31}\right) \\
\mathrm{DMF}^{4}(10) / 145 / 48\end{array}$ & heterogeneous composite powder/4 & $5 \mathrm{mg}$ & TCAs $^{32}(2) /$ plasma \& urine $(6 \mathrm{~mL})$ & $2-4 \mu \mathrm{g} \cdot \mathrm{L}^{-1} /<5.2$ & $\mathrm{LC}^{6}-\mathrm{UV}^{17}$ & [91] \\
\hline
\end{tabular}


Table 2. Cont

\begin{tabular}{|c|c|c|c|c|c|c|}
\hline $\begin{array}{l}\text { MOF (metallic ion \& ligand) } \\
\text { Synthetic solvent }(\mathrm{mL}) / \mathrm{T}\left({ }^{\circ} \mathrm{C}\right) / \text { time }(\mathrm{h})\end{array}$ & $\begin{array}{l}\text { Microextraction format/Extraction } \\
\text { time (min) }\end{array}$ & MOF amount & Analytes (number)/Sample (amount) & $\operatorname{LOD}^{1}\left(\mathrm{ng} \cdot \mathrm{L}^{-1}\right) / \mathrm{RSD}^{2}(\%)$ & Analytical technique & Ref \\
\hline \multicolumn{7}{|c|}{ SPME } \\
\hline $\begin{array}{l}\mathrm{UiO}-66 / \mathrm{MoS}_{2} \\
\left(\mathrm{Zr}^{+} \& \mathrm{H}_{2} \mathrm{bdc}^{3}+\mathrm{MoS}_{2}\right) \\
\mathrm{DMF}^{4}(20) / 120 / 24\end{array}$ & $\begin{array}{l}\text { stainless steel arrow (MOF attached } \\
\text { with epoxy glue)/30 }\end{array}$ & $\begin{array}{l}-\times 25 \mu \mathrm{m} \\
\text { thickness }\end{array}$ & PAHs ${ }^{18}$ (16)/fish (10 mL alkaline extract) & $0.11-1.40 /<8.6$ & HS-SPME ${ }^{33}-$ GC $^{26}$-MS ${ }^{7}$ & [92] \\
\hline $\begin{array}{l}\text { MAF-66 } \\
\left(\mathrm{Zn}^{2+} \& \mathrm{H}_{2} \mathrm{~N}-\mathrm{Htz}^{34}\right) \\
\text { isopropanol }(50) / \mathrm{RT}^{24} / 72\end{array}$ & $\begin{array}{l}\text { stainless steel fiber (layer-by-layer } \\
\text { deposition)/40 }\end{array}$ & $\begin{array}{l}3.0 \mathrm{~cm} \times 15 \mu \mathrm{m} \\
\text { thickness }\end{array}$ & $\begin{array}{l}\text { PAHs } 18 \text { (7)/water, potatoes \& roast pork } \\
\quad(10 \mathrm{~mL} \text { sample or aqueous extract) }\end{array}$ & $0.1-7.5 /<4.2$ & HS-SPME ${ }^{33}-$ GC $^{26}$-FID ${ }^{35}$ & [93] \\
\hline $\begin{array}{l}\text { JUC-Z2 } \\
\left(\mathrm{Ni}^{2+} \& 2,2^{\prime} \text {-bipyridyl) }\right. \\
\mathrm{DMF}^{4}(80) / 80 / 1\end{array}$ & $\begin{array}{l}\text { functionalized fused silica fiber } \\
\text { (in-situ sol-gel method)/40 }\end{array}$ & $\begin{array}{l}-\times 80 \mu \mathrm{m} \\
\text { thickness }\end{array}$ & aromatic amines $(2) /$ urine $(10 \mathrm{~mL})$ & $0.010-0.012 /<7.7$ & HS-SPME ${ }_{7}^{33}$ GC $^{26}$-MS/MS & [94] \\
\hline $\begin{array}{l}\text { MIL-96 } \\
\left(\mathrm{Al}^{+} \& \mathrm{H}_{3} \mathrm{btc}^{36}\right) \\
\text { water }(30) / 200 / 24\end{array}$ & $\begin{array}{l}\text { stainless steel fiber (MOF attached } \\
\text { with epoxy glue)/30 }\end{array}$ & $\begin{array}{l}2.0 \mathrm{~cm} \times 80 \mu \mathrm{m} \\
\text { thickness }\end{array}$ & $\begin{array}{c}\text { THMs }^{37}(4) \text { and TCNM } \\
\mathrm{mL} \text { ) }\end{array}$ & $3.0-11 /<10.1$ & HS-SPME ${ }^{33}-$ GC $^{26}$-MS ${ }^{7}$ & [95] \\
\hline $\begin{array}{l}\mathrm{UiO}-66 \\
\left(\mathrm{Zr}^{4+} \& \mathrm{H}_{2} \mathrm{bdc}^{3}\right) \\
\mathrm{DMF}^{4}(40) / 120 / 24\end{array}$ & $\begin{array}{l}\text { stainless steel fiber (MOF attached } \\
\text { with silicone sealant)/40 }\end{array}$ & $\begin{array}{l}4.0 \mathrm{~cm} \times 8.5 \mu \mathrm{m} \\
\text { thickness }\end{array}$ & $\begin{array}{c}\text { PAHs }{ }^{18}(9) / \text { waters } \\
(10 \mathrm{~mL})\end{array}$ & $10-30 /<5.6$ & HS-SPME ${ }^{33}$-GC ${ }^{26}$-FID ${ }^{35}$ & [96] \\
\hline \multicolumn{7}{|c|}{ SBSME } \\
\hline $\begin{array}{l}\mathrm{UiO}-66-\mathrm{NH}_{2} \\
\left(\mathrm{Zr}^{+4} \& \mathrm{NH}_{2}-\mathrm{H}_{2} \mathrm{bdc}^{20}\right) \\
\mathrm{DMF}^{4}(10) \& \text { acetic acid }(7) / 120 / 24\end{array}$ & $\begin{array}{l}\text { coated glass bar }(\text { in-situ } \\
\text { polymerization method)/30 }\end{array}$ & $\begin{array}{l}1 \mathrm{~cm} \times 4000 \mu \mathrm{m} \\
\text { thickness }\end{array}$ & $\begin{array}{l}\text { SUHs }^{29}(5) / \text { water \& soil (5 mL sample or } \\
\text { aqueous extract) }\end{array}$ & $40-840 /<13.8$ & $\mathrm{LC}^{6}{ }^{6} \mathrm{UV}^{17}$ & [97] \\
\hline $\begin{array}{l}\text { MIL-68@PEEK } \\
\left(\mathrm{Al}^{39} \& \mathrm{P}_{2} \mathrm{H}^{39} \mathrm{bdc}^{3}\right) \\
\mathrm{DMF}^{4}(10) / 130 / 18.5\end{array}$ & $\begin{array}{l}\text { coated dumbbell-shaped bar } \\
\text { (covalent immobilization)/120 }\end{array}$ & $\begin{array}{l}3 \mathrm{~cm} \times 18.4 \mu \mathrm{m} \\
\text { thickness }\end{array}$ & $\begin{array}{l}\text { parabens (3)/creams \& rabbit plasma (20 } \\
\mathrm{mL} \text { aqueous extract) }\end{array}$ & $1-2 /<9.74$ & $\mathrm{LC}^{6}-\mathrm{MS}^{7}$ & [98] \\
\hline $\begin{array}{l}\text { ZIF-67 } \\
\left(\mathrm{Co}^{2+} \& 2-\mathrm{MIm}^{23}\right) \\
\text { methanol }(80) / 120 / 5\end{array}$ & $\begin{array}{l}\text { anodized aluminum bar (in-situ } \\
\text { growth)/20 }\end{array}$ & $\begin{array}{l}1 \mathrm{~cm} \times 500 \mu \mathrm{m} \\
\text { thickness }\end{array}$ & caffeine (1)/beverage \& urine $(10 \mathrm{~mL})$ & $50-100 /<6.1$ & $\mathrm{LC}^{6}{ }^{6} \mathrm{UV}^{17}$ & [99] \\
\hline \multicolumn{3}{|c|}{1 limit of detection } & \multicolumn{2}{|c|}{${ }^{21}$ photodiode array detector } & & \\
\hline \multirow{2}{*}{\multicolumn{3}{|c|}{$\begin{array}{l}2 \text { inter-day relative standard deviation } \\
3 \text { terephthalic acid (benzene-1,4-dicarboxylic acid) }\end{array}$}} & \multirow{2}{*}{\multicolumn{2}{|c|}{22 magnetic graphene }} & & \\
\hline & & & & & & \\
\hline \multicolumn{3}{|c|}{${ }_{5}^{4} N, N$-dimethylformamide } & \multicolumn{2}{|l|}{$\begin{array}{l}23 \\
{ }^{24} \text {-methylimidazole } \\
{ }^{24} \text { room temperature }\end{array}$} & & \\
\hline \multicolumn{3}{|l|}{5 polypropylene } & \multicolumn{2}{|c|}{$\begin{array}{l}{ }^{24} \text { room temperature } \\
25 \text { phthalate esters }\end{array}$} & & \\
\hline \multicolumn{3}{|l|}{${ }^{6}$ liquid chromatography } & \multicolumn{2}{|c|}{26 gas chromatography } & & \\
\hline \multirow{2}{*}{\multicolumn{3}{|c|}{$\begin{array}{l}7 \text { mass spectrometry } \\
8 \text { meso-tetra(4-carboxyphenyl)porphyrin }\end{array}$}} & \multicolumn{2}{|l|}{${ }_{28}^{27}$ graphene oxide } & & \\
\hline & & & \multirow{2}{*}{\multicolumn{2}{|c|}{$\begin{array}{l}28 \text { polydopamine } \\
29 \text { sulfonylurea herbicides }\end{array}$}} & & \\
\hline \multicolumn{3}{|c|}{${ }^{9}$ cold vapor atomic absorption spectroscopy } & & & & \\
\hline \multicolumn{3}{|c|}{10 2-nitroterephthalic acid (2-nitrobenzene-1,4-dicarboxylic acid) } & \multicolumn{2}{|c|}{$\begin{array}{l}29 \text { sulfonylurea herbicides } \\
30 \text { benzodiazepines }\end{array}$} & & \\
\hline \multicolumn{3}{|c|}{$\begin{array}{l}11 \text { endocrine disrupting chemicals } \\
12 \text { diode array detector }\end{array}$} & \multicolumn{2}{|c|}{$314,4^{\prime}$-oxybis(benzoic acid) } & & \\
\hline \multicolumn{3}{|c|}{$\begin{array}{l}{ }^{12} \text { diode array detector } \\
{ }^{13} \text { graphite furnace atomic absorption spectroscopy }\end{array}$} & $\begin{array}{l}32 \text { tricyclic antidepre } \\
33 \text { headspace solid-p }\end{array}$ & 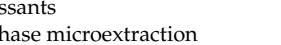 & & \\
\hline 14 1,2,4-triazole & & & 34 -amino-1,2,4-tria & & & \\
\hline${ }_{15}^{15} \mathrm{~N}, \mathrm{~N}$-dimethylacetamide & & & 35 flame ionization d & etector & & \\
\hline${ }_{16}^{16}$ personal care products & & & ${ }^{36}$ trimesic acid (ben & zene-1,3,5-tricarboxylic acid) & & \\
\hline 17 ultraviolet detector & & & 37 trihalomethanes & & & \\
\hline 18 polycyclic aromatic hydrocarbons & & & 38 trichloronitrometl & & & \\
\hline 19 fluorescence detector & & & ${ }^{39}$ polyether ether $\mathrm{ke}$ & & & \\
\hline${ }^{20} 2$-aminoterephthalic acid (2-aminoben & ene-1,4-dicarboxylic acid) & & -: non-reported & & & \\
\hline
\end{tabular}




\subsection{MOFs in Chromatography}

MOFs, and particularly nanoMOFs, are a priori quite interesting materials as chromatographic stationary phases because their pore dimensions make it possible to significantly reduce the amount of required eluents [13]. Their applicability is still lower than that experienced in analytical sample preparation, but increasing studies are currently undertaken. As a trend, the initial studies were mostly with MOFs in gas chromatography (GC), but many MOFs are not stable at the high temperatures required in GC, and nowadays, their inclusion in liquid chromatography (LC) is much higher $[19,20]$. Neat MOFs' permanent porosity is clearly favorable when used as stationary phase in LC, because it permits high flow rates through it with low back-pressure and, ultimately, favors miniaturization. Easy linkage of MOFs to silica and other composites, including their incorporation in monoliths, also favor the applicability of MOFs in LC stationary phases. Furthermore, MOFs designed to present a specific and unique chiral center type are quite attractive to perform chiral separations [100], of the utmost importance in pharmaceutical, industrial and biomedicine applications. In any case, the incorporation of MOFs as stationary phases in GC and LC, or as pseudo-stationary phases in capillary electro-chromatography (CEC), benefits from the low numbers of MOFs required in such phases, together with the clear reutilization of such phases.

Table 3 includes a summary of representative analytical applications of MOFs when included as stationary of pseudo-stationary phases in different chromatographic techniques in the last three years [101-115]. Mostly, MOFs form part of composites with silica or polymers in such phases [101,102], hardly being utilized in its neat appearance. It is important to highlight the number of applications with chiral MOFs (prepared incorporating chiral organic ligands) [108-111].

Green aspects in these applications are from the need of low amounts of MOFs to prepare the phases (from $2 \mathrm{mg}$ of $\mathrm{H}_{2} \mathrm{~N}-\mathrm{UiO}-66$ [103] in a capillary column to $1.5 \mathrm{~g}$ of $\gamma$-CD-MOF [106] in a packed column), reutilization of the as-prepared MOF-based stationary phases, MOF design, and in some cases, even their synthesis.

\subsection{MOFs as Sensors in Spectroscopic and Alectroanalytical Methos}

Many interesting features of MOFs justify their use as sensors in a number of analytical applications. For instance, some MOFs have semiconductor-like properties, and can serve as photoelectric materials [116]. These properties depend in some cases on the metal nature of the MOF, in other cases on the properties imparted by the organic ligands, and in others on the resulting crystal [117]. When incorporated to certain analytical spectroscopic applications, particularly luminescence-based, the MOF design is also important to ensure proper characteristics in the resulting material. In some cases, the luminescence phenomenon is due to the organic ligand of the MOF [118], and in other cases, to the MOF itself (carefully designing the ligand with proper coordination of the ligands to the metal) $[119,120]$. 
Table 3. Representative examples of chromatographic stationary or pseudo-stationary phases incorporating MOFs as novel materials in the period 2017-2019.

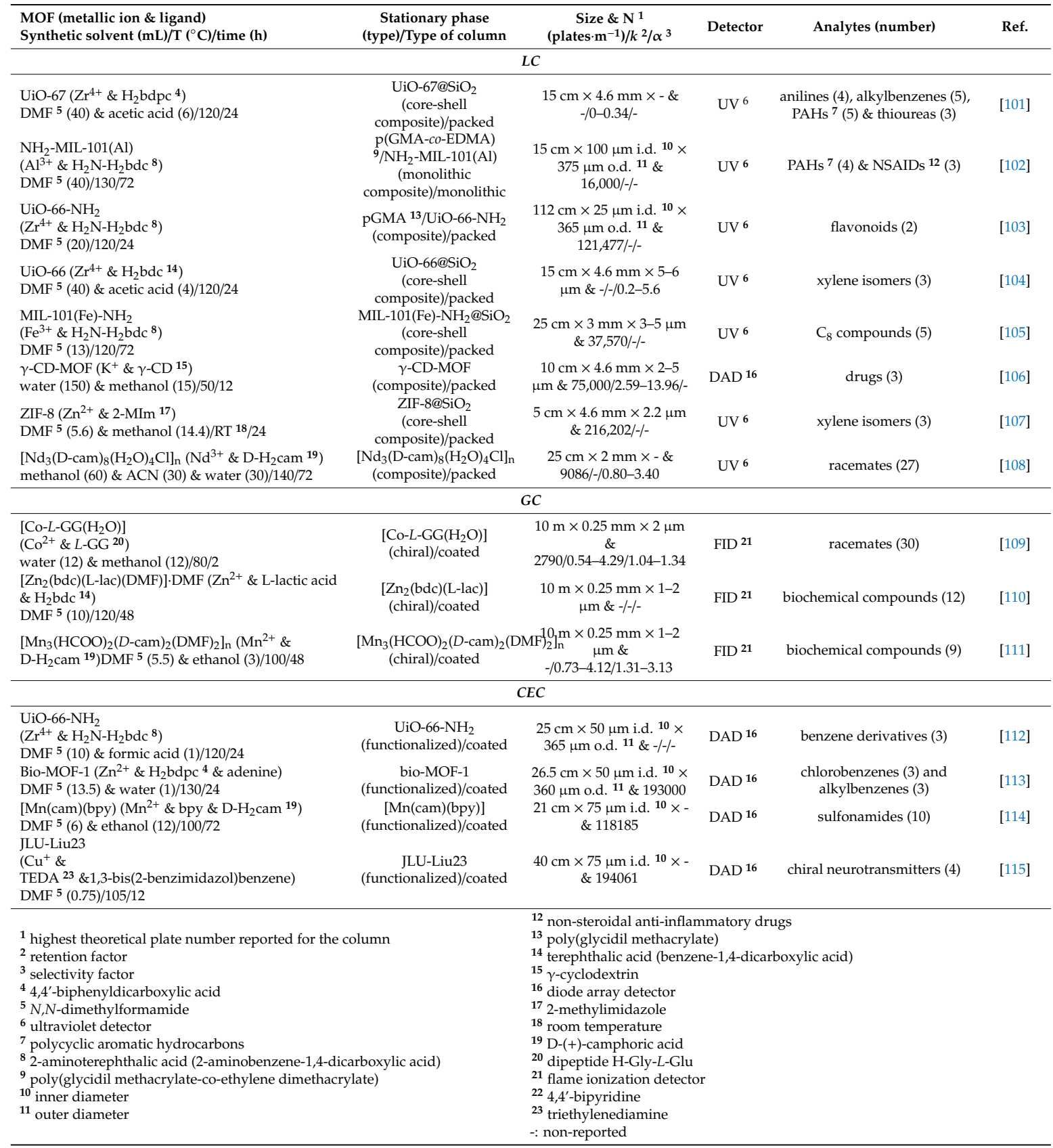

Table 4 lists some representative examples of MOFs used in spectroscopic or in electroanalytical methods in the last three years, together with information of their analytical performance [42,121-128]. 
Table 4. Representative examples of electroanalytical and spectroscopic applications incorporating MOFs in the period 2017-2019.

\begin{tabular}{|c|c|c|c|c|c|c|}
\hline $\begin{array}{l}\text { MOF (metallic ion \& ligand) } \\
\text { Synthetic Solvent }(\mathrm{mL}) / \mathrm{T}\left({ }^{\circ} \mathrm{C}\right) / \text { time }(\mathrm{h})\end{array}$ & MOF-Based Material/amount/comment & Method/Comments & Sample Matrix (amount) & $\operatorname{LOD}^{1 / R_{S D}}{ }^{2}(\%)$ & Analytes (number) & Ref. \\
\hline \multicolumn{7}{|c|}{ Electroanalytical } \\
\hline $\begin{array}{l}\mathrm{ZIF-67} \\
\left(\mathrm{Co}^{2+} \& 2-\mathrm{MIm}^{3}\right) \\
\text { methanol }(100) / \mathrm{RT}^{4} / 24\end{array}$ & neat ZIF- $67 / 43 \mathrm{mg} / \mathrm{CPE}^{5}$ electrode & amperometry/- $50 \mathrm{mV}$ applied & waters (-) & $1.45 \mu \mathrm{M} /<3.26$ & hydrazine (1) & [121] \\
\hline $\begin{array}{l}\text { PCN-224 } \\
\left(\mathrm{Zr}^{+4} \& \mathrm{TCPP} 6\right) \\
\operatorname{DMF}^{7}(50) / 120 / 24\end{array}$ & $\begin{array}{c}\mathrm{PCN} \text { with rGO } 8 / 5 \mathrm{mg} \cdot \mathrm{mL}^{-1} \text { suspension/n-type } \\
\text { semiconductor }\end{array}$ & EIS $9 /-100 \mathrm{mV}$ applied & $\begin{array}{l}\text { water, swine manure \& } \\
\text { lixivium (-) }\end{array}$ & $5.47 \mathrm{ng} \cdot \mathrm{L}^{-1} /<3.8$ & $p$-ASA (1) & [122] \\
\hline $\begin{array}{l}\mathrm{Cu}-\mathrm{MOF} \\
\left(\mathrm{Cu}^{2+} \& \mathrm{H}_{3} \mathrm{btc}^{10}\right) \\
\text { methanol }(12) \& \text { water }(12) / \mathrm{RT}^{4} / 2\end{array}$ & $\begin{array}{l}\text { Cu-MOF with AuNPs } 11 / 60 \mu \mathrm{L} 10 \mu \mathrm{M} \\
\text { solution/aptasensor }\end{array}$ & DPV $^{12} /-300-500 \mathrm{mV}$ applied & $\underset{(-)}{\operatorname{aCSF}}{ }^{13}$ & $0.45 \mathrm{nM} / 1.8$ & $\beta$-amyloid oligomers & [123] \\
\hline $\begin{array}{l}\text { Ni-BTC } \\
\left(\mathrm{Ni}^{2+} \& \mathrm{H}_{3} \mathrm{btc} 10\right) \\
\text { ethanol }(100) \& \text { water }(100) / \mathrm{RT}^{4} / 0.5\end{array}$ & neat Ni-BTC/100 mg/CPE 5 electrode & $\mathrm{CV}^{14} / 400-900 \mathrm{mV}$ applied & soft drinks (-) & $0.08 \mathrm{nM} /<5$ & Ponceau 4R (1) & [117] \\
\hline $\begin{array}{l}\mathrm{H}_{2} \mathrm{~N}-\mathrm{MIL}-53(\mathrm{Al}) \\
\left(\mathrm{A}^{3+} \& \mathrm{H}_{2} \mathrm{~N}-\mathrm{H}_{2} \mathrm{bdc} 15\right) \\
\text { water }(150) / \text { reflux } / 72\end{array}$ & $\mathrm{H}_{2} \mathrm{~N}-\mathrm{MIL}-53(\mathrm{Al})$ in polymeric matrix $/ 40 \mathrm{wt} \% \mathrm{MOF} /$ & EIS $9 / 1000 \mathrm{mV}$ applied & - & - & $\begin{array}{l}\text { methanol (1) \& water (1), (in } \\
\text { gas phase) }\end{array}$ & [124] \\
\hline $\begin{array}{l}\text { Ce-MOF } \\
\left(\mathrm{Ce}^{3+} \& \mathrm{H}_{3} \mathrm{btc}^{10}\right) \\
\text { ethanol (25) \& water }(25) / 90 / 2\end{array}$ & Сe-МОF@MCA 16/1 mg/aptasensor & EIS $\%$ & milk, urine \& water (-) & $17.4 \mathrm{fg} \cdot \mathrm{mL}^{-1} /<2.65$ & ОTC ${ }^{17}(1)$ & [125] \\
\hline $\begin{array}{l}\text { Cu-MOF } \\
\left(\mathrm{Cu}^{2+} \& \text { \& } \mathrm{H}_{3} \mathrm{btc} 10\right) \\
\text { ethanol }(7.1) \& \text { water }(7.1) / 150 / 24\end{array}$ & Cu-MOF-GN $18 / 2 \mathrm{mg} / \mathrm{GCE}{ }^{19}$ electrode & $\begin{array}{c}\mathrm{CV}^{14} \& \mathrm{DPV}{ }^{12} /-0.4-0.8 \mathrm{~V} \& \mathrm{C} \\
-0.2-0.6 \mathrm{~V}\end{array}$ & water (-) & $0.33-0.59 \mu \mathrm{M} /<2.8$ & $\mathrm{HQ}^{20}(1) \& \mathrm{CT}^{21}(1)$ & [126] \\
\hline \multicolumn{7}{|c|}{ Spectroscopic } \\
\hline $\begin{array}{l}\text { Mg-MOF } 1 \\
\left(\mathrm{Mg}^{2+} \& \mathrm{H}_{2} \mathrm{ATDC}^{22}\right) \\
\mathrm{DMF}(4) \text { \& methanol (4) \& water (2)/90/96 }\end{array}$ & neat Mg-MOF 1/-/pH and thermo stability & fluorescence $/ \mathrm{K}_{\mathrm{sv}}{ }^{23}=0.58 \times 10^{4}$ & - & $0.01-0.12 \mu \mathrm{M} /-$ & $\mathrm{Cr}^{3+}(1) \& \operatorname{NAEs}^{24}(8)$ & [118] \\
\hline 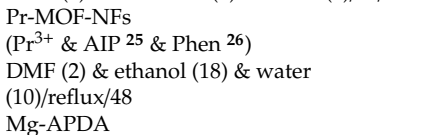 & neat Pr-MOF-NFs ${ }^{27} / 1 \times 10^{-4} \mathrm{M} /$ in distilled water & fluorescence/- & serum (-) & $0.276 \mathrm{ng} \cdot \mathrm{mL}^{-1} /<1.42$ & prolactin (1) & [42] \\
\hline $\begin{array}{l}\left(\mathrm{Mg}^{2+} \& \mathrm{H}_{2} \mathrm{APDA}^{28}\right) \\
\text { DMA } 29(2) \text { \& ethanol (0.1) \& water } \\
(0.1) / 140 / 48\end{array}$ & neat Mg-APDA/2.5 mg/in DMF 7 & fluorescence $/ \mathrm{K}_{\mathrm{sv}}{ }^{23}=2.06 \times 10^{4}$ & - & $126-152 \mu g \cdot L^{-1} /-$ & $\begin{array}{c}\mathrm{Fe}^{3+} \text { (1), pesticides (5) \& } \\
\text { antibiotics (9) }\end{array}$ & [119] \\
\hline $\begin{array}{l}\left.\mathrm{Zr}_{6} \mathrm{O}_{4}(\mathrm{OH})_{4}\left(2,7-\mathrm{CDC}_{6}\right)_{6}\right] \cdot 19 \mathrm{H}_{2} \mathrm{O} \cdot 2 \mathrm{DMF} \\
\left(\mathrm{Zr}^{4+} \& 2,7-\mathrm{H}_{2} \mathrm{CDC} 30\right. \\
\text { \& acetic acid }(0.27) / 80 / 24\end{array}$ & neat Zr-based MOF/2 mg/photostable and reusable & fluorescence $/ \mathrm{K}_{\mathrm{sv}} 23=5.5 \times 10^{4}$ & - & $0.02-0.91 \mu \mathrm{M}$ & $\begin{array}{c}\mathrm{Fe}^{3+}(1), \mathrm{CN}^{-}(1) \& \mathrm{PNP}^{31} \\
\text { (1) }\end{array}$ & [127] \\
\hline $\begin{array}{l}\mathrm{Zn-MOF-1} \\
\left(\mathrm{Zn}^{2+} \& \mathrm{H}_{2} \mathrm{bdc}^{32} \& \mathrm{~L}^{33}\right) \\
\text { water (3) \& ethanol (3)/170/72 }\end{array}$ & neat Zn-MOF-1/-in methanol or water & fluorescence $/ \mathrm{K}_{\mathrm{sv}}{ }^{23}=2.36 \times 10^{4}$ & - & $1.90-3.84 \mu \mathrm{M} /-$ & $\begin{array}{l}\mathrm{Fe}^{3+}(1), 2,6-\text { Dich-4-NA }{ }^{34} \\
\quad \text { (1) \& } \mathrm{Cr}^{6+} \text { ions (2) }\end{array}$ & [128] \\
\hline $\begin{array}{l}\left.\text { Tb-MOF(Tb' } \mathrm{Tb}^{3+} \& \mathrm{AII} 25\right) \\
\text { ethanol }(40) \& \text { water }(40) / \mathrm{RT} / 1\end{array}$ & $\begin{array}{l}\text { Tb-MOF-PMMA } 35 / 50 \mathrm{mg} / \mathrm{MOF} \text { loaded in the } \\
\text { polymeric membrane }\end{array}$ & fluorescence $/ \mathrm{K}_{\mathrm{sv}}{ }^{23}=4.0 \times 10^{4}$ & serum \& water (-) & $0.30-0.35 \mu \mathrm{M} /<4.7$ & $\operatorname{NFAs}^{36}(2)$ & [120] \\
\hline
\end{tabular}


Table 4. Cont.

\begin{tabular}{|c|c|c|c|c|c|}
\hline $\begin{array}{l}\text { MOF (metallic ion \& ligand) } \\
\text { Synthetic Solvent }(\mathrm{mL}) / \mathrm{T}\left({ }^{\circ} \mathrm{C}\right) / \text { time }(\mathrm{h})\end{array}$ & Method/Comments & Sample Matrix (amount) & $\operatorname{LOD}^{1 / R^{\prime}}{ }^{2}(\%)$ & Analytes (number) & Ref. \\
\hline $\begin{array}{l}1 \text { limit of detection } \\
{ }^{2} \text { relative standard deviation } \\
3 \text { 2-methylimidazole } \\
{ }^{4} \text { room temperature } \\
5 \text { carbon paste electrode } \\
6 \text { meso-tetra(4-carboxyphenyl)porphyrin } \\
{ }^{7} \text { N,N-dimethylformamide } \\
{ }^{8} \text { reduced graphene oxide } \\
{ }^{9} \text { electrochemical impedance spectroscopy } \\
10 \text { trimesic acid (benzene-1,3,5-tricarboxylic acid) } \\
11 \text { gold nanoparticles } \\
12 \text { differential pulse voltammetry } \\
13 \text { artificial cerebrospinal fluid } \\
14 \text { cyclic voltammetry } \\
15 \text { 2-aminoterephthalic acid (2-aminobenzene-1,4-dicarboxylic acid) } \\
16 \text { melamine and cyanutic acidmonomers } \\
17 \text { oxytetracycline }\end{array}$ & 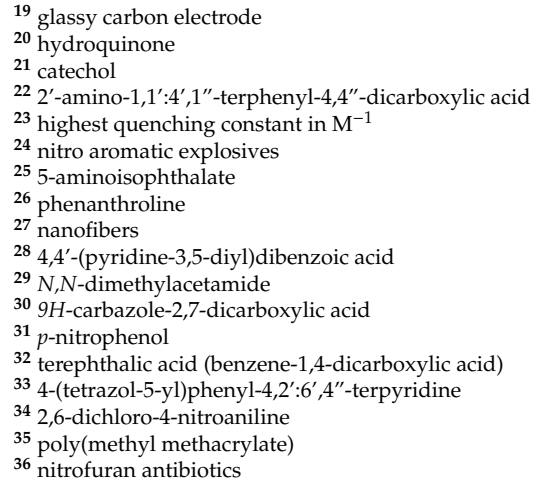 & & & & \\
\hline
\end{tabular}


The reported electroanalytical applications normally perform in electrochemical cells constituted by the electrolyte and three electrodes: a reference electrode, an auxiliary electrode (i.e., $\mathrm{Au} \mathrm{or} \mathrm{Pt)} \mathrm{and}$ the working electrode. MOFs incorporate synthetically in the working electrode by proper modification of the support material, with the purpose of attaining ultra-functional sensors. Thus, Asadi et al. [121] and Ji et al. [117] described carbon paste electrodes (CPE) modified with neat MOFs, such as ZIF-67 and Ni-BTC. MOFs can be combined with nanoparticles with the purpose of improving the overall electrochemical behavior of the resulting composite [121]. Zhou et al. described the use of gold nanoparticles modified with aptamers (to ensure $\beta$-amyloid oligomers detection) and linked to a $\mathrm{Cu}-\mathrm{MOF}$ [123]. Devices that are more complex include not only the neat MOF linked to the working electrode, but also a polymeric matrix as a part of the electrochemical system [124].

Electroanalytical applications with MOFs cover techniques like amperometry [122], differential pulse voltammetry (DPV) [123] and electrochemical impedance spectroscopy (EIS) [125].

It is important to highlight the sensitivity of the MOF-based sensors linked to the electroanalytical applications. Thus, detection limits range from $0.8 \mathrm{nM}$ for Ponceau $4 \mathrm{R}$ in soft drinks (only requiring 43 $\mathrm{mg}$ of MOF) [117] to $1.45 \mu \mathrm{M}$ for hydrazine in waters (in this case requiring $100 \mathrm{mg}$ of MOF) [121]. Furthermore, electroanalytical methods are quite efficient, fast and simple, thus in accordance with GAC. In the reported applications, there is an enormous variety in the nature of the analytes determined, from drugs [126] to peptides [123]; and analyzing quite different samples, like waters [121], bio-fluids [123], and food samples [125].

Spectroscopic applications take advantage of fluorescent MOFs. Luminescent MOFs show prominent optical properties, and relatively long emission wavelength, for which the results are quite advantageous. Furthermore, luminescence sensing has attracted great attention, owing to its high sensitivity, fast response, obvious selectivity, recyclability, and simplicity regarding the instrumentation required which, in this latter case, is also linked to low costs. All these performance characteristics represent very adequate results from a GAC point of view. Regarding additional advantages of minimization of reagents consumption in these luminescence applications, low amounts of MOFs are needed: from $2 \mathrm{mg}$ of neat Zr-based MOF [118] to $50 \mathrm{mg}$ of Tb-MOF-PMMA [120]. Rare earth metals $[42,120]$ and transition metals [127] normally form luminescent MOFs. In new green trends, the interest is tangible in obtaining luminescent MOFs with alkaline-earth metals in their structure $[118,119]$, together with improvements of water stability of the resulting luminescent MOF.

As with electroanalytical applications, luminescent applications cover the determination of organic compounds [42,120], heavy metals [118,127], and even the simultaneous determination of heavy metals and organic compounds [119], as well as with samples like serum [42,120], and water [120].

\section{Concluding Remarks}

MOFs, as materials with almost endless applications in analytical chemistry, must comply with environmental requirements in order to follow properly GAC rules. Thus, assurance of their sustainability must begin with the MOF design (with the proper choice of the MOF constituents), followed by an adequate synthetic procedure and toxicity evaluation of the resulting material, ending up in an analytical method that can be categorized as a GAC method. This, in turn, requires an important collaboration between materials science and analytical chemistry, with an emphasis on green chemistry. Finally, and even more importantly, the rationale behind selecting a MOF for a particular application must always be the first step when setting up an MOF-based analytical method.

Author Contributions: Conceptualization, J.P. and V.P.; Formal analysis, P.R.-B. and I.T.-M.; Funding acquisition, J.P. and V.P.; Investigation, P.R.-B., I.T.-M., J.P. and V.P.; Methodology, P.R.-B., I.T.-M., J.P. and V.P.; Resources, J.P. and V.P.; Supervision, J.P. and V.P.; Writing-original draft, P.R.-B. and I.T.-M.; Writing-review \& editing, J.P. and V.P.

Funding: V.P. thanks the Project Ref. MAT2017-89207-R.

Acknowledgments: I.T.-M. thanks his collaboration fellowship with the Spanish Ministry of Education (MEC) during the MS studies at ULL. J.P. thanks the "Agustín de Betancourt" Canary Program for his research associate 
position at ULL. V.P. acknowledges the Spanish Ministry of Economy and Competitiveness (MINECO) for the Project Ref. MAT2017-89207-R.

Conflicts of Interest: The authors declare no conflict of interest.

\section{References}

1. Matsuyama, S.; Munakata, M.; Emori, T.; Kitagawa, S. Synthesis and Crystal Structures of Novel One-dimensional - Polymers, $\left[\left\{\mathrm{M}(\right.\right.$ bpen $\left.) \mathrm{X}_{\infty}\right\}, 1\left[\mathrm{M}=\mathrm{Cu}^{\prime}, \mathrm{X}=\mathrm{PF}_{6}^{-} ; \mathrm{M}=\mathrm{Ag}^{\prime}, \mathrm{X}=\mathrm{ClO}_{4^{-}}\right.$; bpen $=$ trans-I,2-bis (2-pyridyl)ethylene $]$ and $\left[\left\{\mathrm{Cu}(\text { bpen })(\mathrm{CO})\left(\mathrm{CH}_{3} \mathrm{CN}\right)\left(\mathrm{PF}_{6}\right)\right\}_{\infty}\right]$. J. Chem. Soc. Dalton Trans. 1991, 1, 2869-2874.

2. Yaghi, O.M.; Hailian, L. Hydrothermal Synthesis of a Metal-Organic Framework Containing Large Rectangular Channels. J. Am. Chem. Soc. 1995, 117, 10401-10402. [CrossRef]

3. Riou, D.; Férey, G. Hybrid open frameworks (MIL-n). Part 3: Crystal structures of the HT and LT forms of MIL-7: A new vanadium propylenediphosphonate with an open-framework. Influence of the synthesis temperature on the oxidation state of vanadium within the same structural type. J. Mater. Chem. 1998, 8, 2733-2735.

4. Farha, O.K.; Eryazici, I.; Jeong, N.C.; Hauser, B.G.; Wilmer, C.E.; Sarjeant, A.A.; Snurr, R.Q.; Nguyen, S.B.T.; Yazaydın, A.Ö.; Hupp, J.T. Metal-Organic Framework Materials with Ultrahigh Surface Areas: Is the Sky the Limit? J. Am. Chem. Soc. 2012, 134, 15016-15021. [CrossRef]

5. Khan, N.A.; Hasan, Z.; Jhung, S.H. Adsorptive removal of hazardous materials using metal-organic frameworks (MOFs): A review. J. Hazard. Mater. 2013, 244-245, 444-456. [CrossRef]

6. Moghadam, P.Z.; Li, A.; Wiggin, S.B.; Tao, A.; Maloney, A.G.P.; Wood, P.A.; Ward, S.C.; Fairen-Jimenez, D. Development of a Cambridge Structural Database Subset: A Collection of Metal-Organic Frameworks for Past, Present, and Future. Chem. Mater. 2017, 29, 2618-2625. [CrossRef]

7. Stavila, V.; Talin, A.A.; Allendorf, M.D. MOF-based electronic and opto-electronic devices. Chem. Soc. Rev. 2014, 43, 5994-6010. [CrossRef]

8. Bae, Y.S.; Snurr, R.Q. Development and evaluation of porous materials for carbon dioxide separation and capture. Angew. Chem. Int. Ed. 2011, 50, 11586-11596. [CrossRef]

9. Li, H.; Wang, K.; Sun, Y.; Lollar, C.T.; Li, J.; Zhou, H.-C. Recent advances in gas storage and separation using metal-organic frameworks. Mater. Today 2018, 21, 108-121. [CrossRef]

10. Ma, S.; Zhou, H.-C. Gas storage in porous metal-organic frameworks for clean energy applications. Chem. Commun. 2010, 46, 44-53. [CrossRef]

11. Kang, Y.-S.; Lu, Y.; Chen, K.; Zhao, Y.; Wang, P.; Sun, W.Y. Metal-organic frameworks with catalytic centers: From synthesis to catalytic application. Coord. Chem. Rev. 2019, 378, 262-280. [CrossRef]

12. Abánades-Lázaro, I.; Forgan, R.S. Application of zirconium MOFs in drug delivery and biomedicine. Coord. Chem. Rev. 2019, 380, 230-259. [CrossRef]

13. Pacheco-Fernández, I.; González-Hernández, P.; Pasán, J.; Ayala, J.H.; Pino, V. The rise of metal-organic frameworks in analytical chemistry. In Handbook of Smart Materials in Analytical Chemistry; De la Guardia, M., Esteve-Turrillas, F.A., Eds.; Wiley: Hoboken, NJ, USA, 2016; Volume 1, pp. 463-502.

14. Rocío-Bautista, P.; Pacheco-Fernández, I.; Pasán, J.; Pino, V. Are metal-organic frameworks able to provide a new generation of solid-phase microextraction coatings?-A review. Anal. Chim. Acta 2016, 939, $26-41$. [CrossRef]

15. Rocío-Bautista, P.; González-Hernández, P.; Pino, V.; Pasán, J.; Afonso, A.M. Metal-organic frameworks as novel sorbents in dispersive-based microextraction approaches. Trends Anal. Chem. 2017, 90, 114-134. [CrossRef]

16. Maya, F.; Cabello, C.P.; Frizzarin, R.M.; Estela, J.M.; Palomino, G.T.; Cerdà, V. Magnetic solid-phase extraction using metal-organic frameworks (MOFs) and their derived carbons. Trends Anal. Chem. 2017, 90, 142-152. [CrossRef]

17. Wang, X.; Ye, N. Recent advances in metal-organic frameworks and covalent organic frameworks for sample preparation and chromatographic analysis. Electrophoresis 2017, 38, 3059-3078. [CrossRef]

18. Hashemi, B.; Zohrabi, P.; Raza, N.; Kim, K.-H. Metal-organic frameworks as advanced sorbents for the extraction and determination of pollutants from environmental, biological, and food media. Trends Anal. Chem. 2017, 97, 65-82. [CrossRef] 
19. Maya, F.; Cabello, C.P.; Figuerola, A.; Palomino, G.T.; Cerdà, V. Immobilization of Metal-Organic Frameworks on Supports for Sample Preparation and Chromatographic Separation. Chromatographia 2019, 82, 361-375. [CrossRef]

20. Zhang, J.; Chen, Z. Metal-organic frameworks as stationary phase for application in chromatographic separation. J. Chromatogr. A 2017, 1530,1-18. [CrossRef]

21. Xiaoxin, L.; Lun, S.; Sha, C. Application of Metal-Organic Frameworks in Chromatographic Separation. Acta Chim. Sin. 2016, 74, 969-979.

22. Li, A.; Liu, X.; Chai, H.; Huang, Y. Recent advances in the construction and analytical applications of metal-organic frameworks-based nanozymes. Trends Anal. Chem. 2018, 105, 391-403. [CrossRef]

23. Feldblyum, J.I.; Keenan, E.A.; Matzger, A.J.; Maldonado, S. Photoresponse Characteristics of Archetypal Metal-Organic Frameworks. J. Phys. Chem. C 2012, 116, 3112-3121. [CrossRef]

24. Yadav, D.K.; Ganesan, V.; Marken, F.; Gupta, R.; Sonkar, P.K. Metal@MOF Materials in Electroanalysis: Silver-Enhanced Oxidation Reactivity Towards Nitrophenols Adsorbed into a Zinc Metal Organic Framework-Ag@MOF-5(Zn). Electrochim. Acta 2016, 219, 482-491. [CrossRef]

25. Filippou, O.; Bitas, D.; Samanidou, V. Green approaches in sample preparation of bioanalytical samples prior to chromatographic analysis. J. Chromatogr. B 2017, 1043, 44-62. [CrossRef]

26. Sheldon, R.A. Fundamentals of green chemistry: Efficiency in reaction design. Chem. Soc. Rev. 2012, 41, 1437-1451. [CrossRef]

27. Ludwig, J.K. Green Chemistry: An Introductory Text. Green Chem. Lett. 2017, 10, 30-31. [CrossRef]

28. Valcárcel, M. Principles of Analytical Chemistry; Springer: Berlin, Germany, 2000; ISBN 978-3-642-57157-2.

29. Koel, M.; Kaljurand, M. Green Analytical Chemistry; The Royal Society of Chemistry: Cambridge, UK, 2010; ISBN 978-1-84755-872-5.

30. Namieśnik, J. Trends in Environmental Analytics and Monitoring. Crit. Rev. Anal. Chem. 2000, 30, $221-269$. [CrossRef]

31. Anastas, P.T.; Warner, J.C. Green Chemistry: Theory and Practice; Oxford University Press: New York, NY, USA, 1998; p. 30.

32. Gałuszka, A.; Migaszewski, Z.; Namieśnik, J. The 12 principles of green analytical chemistry and the SIGNIFICANCE mnemonic of green analytical practices. Trends Anal. Chem. 2013, 50, 78-84. [CrossRef]

33. Płotka-Wasylka, J.; Szczepańska, N.; de la Guardia, M.; Namieśnik, J. Miniaturized solid-phase extraction techniques. Trends Anal. Chem. 2015, 73, 19-38. [CrossRef]

34. Tobiszewski, M.; Namieśnik, J. Greener organic solvents in analytical chemistry. Curr. Opin. Green Sust. Chem. 2017, 5, 1-4. [CrossRef]

35. Pacheco-Fernández, I.; Pino, V. Green solvents in analytical chemistry. Curr. Opin. Green Sustain. Chem. 2019, 18, 42-50. [CrossRef]

36. Yaghi, O.M.; O'Keeffe, M.; Ockwig, N.W.; Chae, H.K.; Eddaoudi, M.; Kim, J. Reticular synthesis and the design of new materials. Nature 2003, 423, 705-715. [CrossRef]

37. Furukawa, H.; Cordova, K.E.; O'Keeffe, M.; Yaghi, O.M. The Chemistry and Applications of Metal-Organic Frameworks. Science 2013, 341, 12304441-123044412. [CrossRef]

38. Bai, Y.; Dou, Y.; Xie, L.-H.; Rutledge, W.; Li, J.-R.; Zhou, H.-C. Zr-based metal-organic frameworks: Design, synthesis, structure, and applications. Chem. Soc. Rev. 2016, 45, 2327-2367. [CrossRef]

39. Rajkumar, T.; Kukkar, D.; Kim, K.-H.; Sohn, J.R.; Deed, A. Cyclodextrin-metal-organic framework (CD-MOF): From synthesis to applications. J. Ind. Eng. Chem. 2019, 72, 50-66. [CrossRef]

40. Gaab, M.; Trukhan, N.; Maurer, S.; Gummaraju, R.; Müller, U. The progression of Al-based metal-organic frameworks-From academic research to industrial production and applications. Micropor. Mesopor. Mater. 2012, 157, 131-136. [CrossRef]

41. Kim, J.; Lee, S.; Kim, J.; Lee, D. Metal-organic frameworks derived from zero-valent metal substrates: Mechanisms of formation and modulation of properties. Adv. Funct. Mater. 2019, 29, 1808466. [CrossRef]

42. Sheta, S.M.; El-Sheikh, S.M.; Abd-Elzaher, M.M. A novel optical approach for determination of prolactin based on Pr-MOF nanofibers. Anal. Bioanal. Chem. 2019. [CrossRef]

43. Reinsch, H. “Green” Synthesis of Metal-Organic Frameworks. Eur. J. Inorg. Chem. 2016, 27, 4290-4299. [CrossRef]

44. Rubio-Martinez, M.; Avci-Camur, C.; Thornton, A.W.; Imaz, I.; Maspoch, D.M.; Hill, M.R. New synthetic routes towards MOF production at scale. Chem. Soc. Rev. 2017, 46, 3453-3480. [CrossRef] 
45. Klimakow, M.; Klobes, P.; Thunemann, A.F.; Rademann, K.; Emmerling, F. Mechanochemical synthesis of Metal-Organic Frameworks: A fast and facile approach toward quantitative yields and high specific surface areas. Chem. Mater. 2010, 22, 5216-5221. [CrossRef]

46. Joaristi, A.M.; Juan-Alcañiz, J.; Serra-Crespo, P.; Kapteijn, F.; Gascón, J. Electrochemical synthesis of some archetypical $\mathrm{Zn}^{2+}, \mathrm{Cu}^{2+}$, and $\mathrm{Al}^{3+}$ Metal Organic Frameworks. Cryst. Growth Des. 2012, 12, 3489-3498. [CrossRef]

47. Julien, P.A.; Mottillo, C.; Friscic, T. Metal-Organic Frameworks meet scalable and sustainable synthesis. Green Chem. 2017, 19, 2729-2747. [CrossRef]

48. Li, P.; Cheng, F.F.; Xiong, W.W.; Zhang, Q.C. New synthetic strategies to prepare metal-organic frameworks. Inorg. Chem. Front. 2018, 5, 2693-2708. [CrossRef]

49. Khan, N.A.; Jhung, S.-H. Synthesis of Metal-Organic Frameworks (MOFs) with microwave or ultrasound: Rapid reaction, phase selectivity, and size reduction. Coord. Chem. Rev. 2015, 285, 11-23. [CrossRef]

50. Sánchez-Sánchez, M.; Getachew, N.; Díaz, K.; Díaz-García, M.; Chebude, Y.; Díaz, I. Synthesis of metal-organic frameworks in water at room temperature: Salts as linker sources. Green Chem. 2015, 17, 1500-1509. [CrossRef]

51. Zhang, J.; Bu, J.T.; Chen, S.; Wu, T.; Zheng, S.; Chen, Y.; Nieto, R.A.; Feng, P.; Bu, X. Urothermal synthesis of crystalline porous materials. Angew. Chem. Int. Ed. 2010, 49, 8876-8879. [CrossRef]

52. Tranchemontagne, D.J.; Hunt, J.R.; Yaghi, O.M. Room temperature synthesis of metal-organic frameworks: MOF-5, MOF-74, MOF-177, MOF-199, and IRMOF-0. Tetrahedron 2008, 64, 8553-8557. [CrossRef]

53. Bayliss, P.A.; Ibarra, I.A.; Pérez, E.; Yang, S.; Tang, C.C.; Poliakoff, M.; Schröder, M. Synthesis of metal-organic frameworks by continuous flow. Green Chem. 2014, 16, 3796-3802. [CrossRef]

54. Rocío-Bautista, P.; Pino, V.; Ayala, J.H.; Ruiz-Pérez, C.; Vallcorba, O.; Afonso, A.M.; Pasán, J. A green metal-organic framework to monitor water contaminants. RSC Adv. 2018, 8, 31304-31310. [CrossRef]

55. Noh, H.; Kung, C.-W.; Islamoglu, T.; Peters, A.W.; Liao, Y.; Li, P.; Garibay, S.J.; Zhang, X.; DeStefano, M.R.; Hupp, J.T.; et al. Room Temperature Synthesis of an 8-Connected Zr-Based Metal-Organic Framework for Top-Down Nanoparticle Encapsulation. Chem. Mater. 2018, 30, 2193-2197. [CrossRef]

56. Lenstra, D.C.; Rutjes, F.P.J.T. Organic synthesis in flow: Toward higher levels of sustainability. In Sustainable Flow Chemistry: Methods and Applications; Vaccaro, L., Ed.; Wiley: Hoboken, NJ, USA, 2017; pp. 103-134.

57. Leardi, R. Experimental design in chemistry: A tutorial. Anal. Chim. Acta 2009, 652, 161-172. [CrossRef]

58. Tamames-Tabar, C.; Cunha, D.; Imbuluzqueta, E.; Ragon, F.; Serre, C.; Blanco-Prieto, C.M.; Horcajada, P. Cytotoxicity of nanoscaled metal-organic frameworks. J. Mater. Chem. B 2014, 2, 262-271. [CrossRef]

59. Chen, G.; Leng, X.; Luo, J.; You, L.; Qu, C.; Dong, X.; Huang, H.; Yin, X.; Ni, J. In Vitro Toxicity Study of a Porous Iron (III) Metal-Organic Framework. Molecules 2019, 24, 1211. [CrossRef]

60. Horcajada, P.; Chalati, T.; Serre, C.; Gille, B.; Sebrie, C.; Baati, T.; Eubank, J.F.; Heurtau, D.; Clayette, P.; Kreuz, C.; et al. Porous metal-organic-framework nanoscale carriers as a potential platform for drug delivery and imaging. Nat. Mater. 2010, 9, 172-179. [CrossRef]

61. Su, F.; Jia, Q.; Li, Z.; Wang, M.; He, L.; Peng, D.; Song, Y.; Zhang, Z.; Fang, S. Aptamer-templated silver nanoclusters embedded in zirconium metal-organic framework for targeted antitumor drug delivery. Micropor. Mesopor. Mater. 2019, 275, 152-162. [CrossRef]

62. Vinogradov, V.V.; Drozdov, A.S.; Mingabudinova, L.R.; Shabanova, E.M.; Kolchina, N.O.; Anastasova, E.I.; Markova, A.A.; Shtil, A.A.; Milichko, V.A.; Starova, G.L.; et al. Composites based on heparin and MIL-101(Fe): The drug releasing depot for anticoagulant therapy and advanced medical nanofabrication. J. Mater. Chem. B 2018, 6, 2450-2459. [CrossRef]

63. Ma, A.; Luo, Z.; Gu, C.; Li, B.; Liu, J. Cytotoxicity of a metal-organic framework: Drug delivery. Inorg. Chem. Commun. 2017, 77, 68-71. [CrossRef]

64. Cheplakova, A.M.; Solovieva, A.O.; Pozmogova, T.N.; Vorotnikov, Y.A.; Brylev, K.A.; Vorotnikova, N.A.; Vorontsova, E.V.; Mironov, Y.V.; Poveshchenko, A.F.; Kovalenko, A.K.; et al. Nanosized mesoporous metal-organic framework MIL-101 as a nanocarrier for photoactive hexamolybdenum cluster compounds. J. Inorg. Biochem. 2017, 166, 100-107. [CrossRef]

65. Zheng, H.; Zhang, Y.; Liu, L.; Wan, W.; Guo, P.; Nyström, A.M.; Zou, X. One-pot Synthesis of Metal-Organic Frameworks with Encapsulated Target Molecules and Their Applications for Controlled Drug Delivery. J. Am. Chem. Soc. 2016, 138, 962-968. [CrossRef] 
66. Sifaoui, I.; López-Arencibia, A.; Martín-Navarro, C.M.; Reyes-Batlle, M.; Wagner, C.; Chiboub, O.; Mejri, M.; Valladares, B.; Abderrabba, M.; Piñero, J.E.; et al. Programmed cell death in Acanthamoeba castellanii Neff induced by several molecules present in olive leaf extracts. PLOS ONE 2017, 12, 1-12. [CrossRef] [PubMed]

67. Qian, L.; Lei, D.; Duan, X.; Zhang, S.; Song, W.; Hou, C.; Tang, R. Design and preparation of metal-organic framework papers with enhanced mechanical properties and good antibacterial capacity. Carbohydr. Polym. 2018, 192, 44-51. [CrossRef] [PubMed]

68. Zhu, W.; Zhang, L.; Yang, Z.; Liu, P.; Wang, J.; Cao, J.; Shen, A.; Xu, Z.; Wang, J. An efficient tumor-inducible nanotheranostics for magnetic resonance imaging and enhanced photodynamic therapy. Chem. Eng. J. 2019, 358, 969-979. [CrossRef]

69. Xin, X.-T.; Cheng, J.-Z. A mixed-ligand approach for building a N-rich porous metal-organic framework for drug release and anticancer activity against oral squamous cell carcinoma. J. Coord. Chem. 2018, 71, 3565-3574. [CrossRef]

70. Lázaro, I.A.; Haddad, S.; Rodrigo-Muñoz, J.M.; Marshall, R.J.; Sastre, B.; del Pozo, V.; Fairen-Jimenez, D.; Forgan, R.S. Surface-Functionalization of Zr-Fumarate MOF for Selective Cytotoxicity and Immune System Compatibility in Nanoscale Drug Delivery. ACS Appl. Mater. Interf. 2018, 10, 31146-31157. [CrossRef] [PubMed]

71. Shen, S.; Li, L.; Li, S.; Bai, Y.; Liu, H. Metal-organic frameworks induce autophagy in mouse embryonic fibroblast cells. Nanoscale 2018, 10, 18161-18168. [CrossRef] [PubMed]

72. Armenta, S.; Garrigues, S.; Esteve-Turrillas, F.A.; Guardia, M. Green extraction techniques in green analytical chemistry. Trends Anal. Chem. 2019. [CrossRef]

73. Pacheco-Fernández, I.; González-Hernández, P.; Rocío-Bautista, P.; Trujillo-Rodríguez, M.J.; Pino, V. Main uses of Microwaves and Ultrasounds in Analytical Extraction Schemes: An Overview. In Analytical Separation Science; Anderson, J.L., Stalcup, A., Berthod, A., Pino, V., Eds.; Wiley: Hoboken, NJ, USA, 2016; Volume 5, pp. 1469-1501.

74. Yamini, Y.; Rezazadeh, M.; Seidi, S. Liquid-phase microextraction-The different principles and configurations. Trends Anal. Chem. 2019, 112, 264-272. [CrossRef]

75. Mogaddam, M.R.A.; Mohebbi, A.; Pazhohan, A.; Khodadadeian, F.; Farajzadeh, M.A. Headspace mode of liquid phase microextraction: A review. Trends Anal. Chem. 2019, 110, 8-14. [CrossRef]

76. Chisvert, A.; Cárdenas, S.; Lucena, R. Dispersive micro-solid phase extraction. Trends Anal. Chem. 2019, 112, 224-247. [CrossRef]

77. Olcer, Y.A.; Tascon, M.; Eroglu, A.E.; Boyac1, E. Thin film microextraction: Towards faster and more sensitive microextraction. Trends Anal. Chem. 2019, 113, 97-101. [CrossRef]

78. Llompart, M.; Celeiro, M.; García-Jares, C.; Dagnac, T. Environmental applications of solid-phase microextraction. Trends Anal. Chem. 2019, 112, 224-247. [CrossRef]

79. Gao, G.; Xing, X.; Liu, T.; Wang, J.; Hou, X. UiO-66(Zr) as sorbent for porous membrane protected micro-solid-phase extraction androgens and progestogens in environmental water samples coupled with LC-MS/MS analysis: The application of experimental and molecular simulation method. Microchem. J. 2019, 146, 126-133. [CrossRef]

80. Kahkha, M.R.R.; Daliran, S.; Oveisi, A.R.; Kaykhaii, M.; Sepehri, Z. The Mesoporous Porphyrinic Zirconium Metal-Organic Framework for Pipette-Tip Solid-Phase Extraction of Mercury from Fish Samples Followed by Cold Vapor Atomic Absorption Spectrometric Determination. Food Anal. Methods. 2017, 10, 2175-2184. [CrossRef]

81. Gao, G.; Li, S.; Li, S.; Wang, Y.; Zhao, P.; Zhang, X.; Hou, X. A combination of computational-experimental study on metal-organic frameworks MIL-53(Al) as sorbent for simultaneous determination of estrogens and glucocorticoids in water and urine samples by dispersive micro-solid-phase extraction coupled to UPLC-MS/MS. Talanta 2018, 180, 358-367. [CrossRef] [PubMed]

82. Taima-Mancera, I.; Rocío-Bautista, P.; Pasán, J.; Ayala, J.H.; Ruiz-Pérez, C.; Afonso, A.M.; Lago, A.B.; Pino, V. Influence of ligand functionalization of UiO-66-based Metal-Organic Frameworks when used as sorbents in dispersive solid-phase analytical microextraction for different aqueous organic pollutants. Molecules 2018, 23, 2869. [CrossRef]

83. Kashanaki, R.; Ebrahimzadeh, H.; Moradi, M. Metal-organic framework based micro solid phase extraction coupled with supramolecular solvent microextraction to determine copper in water and food samples. New J. Chem. 2018, 42, 5806-5813. [CrossRef] 
84. González-Hernández, P.; Lago, A.B.; Pasán, J.; Ruiz-Pérez, C.; Ayala, J.H.; Afonso, A.M.; Pino, V. Application of a pillared-layer Zn-triazolate metal-organic framework in the dispersive miniaturized solid-phase extraction of personal care products from wastewater samples. Molecules 2019, 24, 690. [CrossRef]

85. Boontongto, T.; Siriwong, K.; Burakham, R. Amine-Functionalized Metal-Organic Framework as a New Sorbent for Vortex-Assisted Dispersive Micro-Solid Phase Extraction of Phenol Residues in Water Samples Prior to HPLC Analysis: Experimental and Computational Studies. Chromatographia 2018, 81, 735-747. [CrossRef]

86. Huang, Y.-F.; Liu, Q.-H.; Li, K.; Li, Y.; Chang, N. Magnetic iron(III)-based framework composites for the magnetic solid-phase extraction of fungicides from environmental water samples. J. Sep. Sci. 2018, 41, 1129-1137. [CrossRef]

87. Lu, Y.; Wang, B.; Yan, Y.; Liang, H.; Wu, D. Silica Protection-Sacrifice Functionalization of Magnetic Graphene with a Metal-Organic Framework (ZIF-8) to Provide a Solid-Phase Extraction Composite for Recognization of Phthalate Easers from Human Plasma Samples. Chromatographia 2019, 82, 625-634. [CrossRef]

88. Liu, G.; Huang, X.; Lu, M.; Li, L.; Li, T.; Xu, D. Facile synthesis of magnetic zinc metal-organic framework for extraction of nitrogen-containing heterocyclic fungicides from lettuce vegetable samples. J. Sep. Sci. 2019, 1, 1-8. [CrossRef] [PubMed]

89. Deng, Y.; Zhang, R.; Li, D.; Sun, P.; Su, P.; Yang, Y. Preparation of iron-based MIL-101 functionalized polydopamine@ $\mathrm{Fe}_{3} \mathrm{O}_{4}$ magnetic composites for extracting sulfonylurea herbicides from environmental water and vegetable samples. J. Sep. Sci. 2018, 41, 2046-2055. [CrossRef] [PubMed]

90. Zhang, S.; Yao, W.; Fu, D.; Zhang, C.; Zhao, H. Fabrication of magnetic zinc adeninate metal-organic frameworks for the extraction of benzodiazepines from urine and wastewater. J. Sep. Sci. 2018, 41, 1864-1870. [CrossRef] [PubMed]

91. Safari, M.; Shahlaei, M.; Yamini, Y.; Shakorian, M.; Arkan, E. Magnetic framework composite as sorbent for magnetic solid phase extraction coupled with high performance liquid chromatography for simultaneous extraction and determination of tricyclic antidepressants. Anal. Chim. Acta 2018, 1034, 204-213. [CrossRef] [PubMed]

92. Yuan, Y.; Lin, X.; Li, T.; Pang, T.; Dong, Y.; Zhuo, R.; Wang, Q.; Cao, Y.; Gan, N. A solid phase microextraction Arrow with zirconium metal-organic framework/molybdenum disulfide coating coupled with gas chromatography-mass spectrometer for the determination of polycyclic aromatic hydrocarbons in fish samples. J. Chromatogr. A 2019, 1592, 9-18. [CrossRef] [PubMed]

93. Liu, M.; Liu, J.; Guo, C.; Li, Y. Metal azolate framework-66-coated fiber for headspace solid-phase microextraction of polycyclic aromatic hydrocarbons. J. Chromatogr. A 2019, 1592, 57-63. [CrossRef] [PubMed]

94. Niu, J.; Zhao, X.; Jin, Y.; Yang, G.; Li, Z.; Wang, J.; Zhao, R.; Li, Z. Determination of aromatic amines in the urine of smokers using a porous organic framework (JUC-Z2)-coated solid-phase microextraction fiber. J. Chromatogr. A 2018, 1555, 37-44. [CrossRef]

95. Zhang, B.; Xu, G.; Li, L.; Wang, X.; Li, N.; Zhao, R.-S.; Lin, J. Facile fabrication of MIL-96 as coating fiber for solid-phase microextraction of trihalomethanes and halonitromethanes in water samples. Chem. Eng. J. 2018, 350, 240-247. [CrossRef]

96. Tian, Y.; Sun, M.; Wang, X.; Luo, C.; Feng, J. A Nanospherical Metal-Organic Framework UiO-66 for Solid-Phase Microextraction of Polycyclic Aromatic Hydrocarbons. Chromatographia 2018, 81, $1053-1061$. [CrossRef]

97. Yang, J.-H.; Cui, C.-X.; Qu, L.-B.; Chen, J.; Zhou, X.-M.; Zhang, Y.-P. Preparation of a monolithic magnetic stir bar for the determination of sulfonylurea herbicides coupled with HPLC. Microchem. J. 2018, 141, 369-376. [CrossRef]

98. Wang, C.; Zhou, W.; Liao, X.; Wang, X.; Chen, Z. Covalent immobilization of metal organic frameworks onto chemical resistant poly(ether ether ketone) jacket for stir bar extraction. Anal. Chim. Acta 2018, 1025, 124-133. [CrossRef] [PubMed]

99. Ghani, M.; Ghoreishi, S.M.; Azamati, M. In-situ growth of zeolitic imidazole framework-67 on nanoporousanodized aluminum bar as stir-bar sorptive extraction sorbent fordetermining caffeine. J. Chromatogr. A 2018, 1577, 15-23. [CrossRef] [PubMed]

100. Bhattacharjee, S.; Khan, M.I.; Li, X.; Zhu, Q.-L.; Wu, X.-T. Recent Progress in Asymmetric Catalysis and Chromatographic Separation by Chiral Metal-Organic Frameworks. Catalysts 2018, 8, 120-148. [CrossRef] 
101. Li, X.; Li, B.; Liu, M.; Zhou, Y.; Zhang, L.; Qiao, X. Core-Shell Metal-Organic Frameworks as the Mixed-Mode Stationary Phase for Hydrophilic Interaction/Reversed-Phase Chromatography. ACS Appl. Mater. Interf. 2019, 11, 10320-10327. [CrossRef] [PubMed]

102. Pérez-Cejuela, H.M.; Carrasco-Correa, E.J.; Shahat, A.; Simó-Alfonso, E.F.; Herrero-Martínez, J.M. Incorporation of metal-organic framework amino-modified MIL-101 into glycidyl methacrylate monoliths for nano LC Separation. J. Sep. Sci. 2019, 42, 834-842. [CrossRef] [PubMed]

103. Chen, K.; Zhang, L.; Zhang, W. Preparation and evaluation of open-tubular capillary column combining a metal-organic framework and a brush-shaped polymer for liquid chromatography. J. Sep. Sci. 2018, 41, 2347-2353. [CrossRef] [PubMed]

104. Gao, B.; Huang, M.; Zhang, Z.; Yang, Q.; Su, B.; Yang, Y.; Ren, Q.; Bao, Z. Hybridization of metal-organic framework and monodisperse spherical silica for chromatographic separation of xylene isomers. Chin. J. Chem. Eng. 2019, 27, 818-826. [CrossRef]

105. Ehrling, S.; Kutzscher, C.; Freund, P.; Müller, P.; Senkovska, I.; Kaskel, S. MOF@SiO 2 core-shell composites as stationary phase in high performance liquid chromatography. Micropor. Mesopor. Mat. 2018, 263, 268-274. [CrossRef]

106. Xu, X.; Wang, C.; Li, H.; Li, X.; Liu, B.; Singh, V.; Wang, S.; Sun, L.; Gref, R.; Zhan, J. Evaluation of drug loading capabilities of $\gamma$-cyclodextrin-metal organic frameworks by high performance liquid chromatography. J. Chromatogr. A 2017, 1488, 37-44. [CrossRef]

107. Qu, Q.; Xuan, H.; Zhang, K.; Chen, X.; Ding, Y.; Feng, S.; Xu, Q. Core-shell silica particles with dendritic pore channels impregnated with zeolite imidazolate framework-8 for high performance liquid chromatography separation. J. Chromatogr. A 2017, 1505, 63-68. [CrossRef] [PubMed]

108. Zhang, P.; Wang, L.; Zhang, J.-H.; He, Y.-J.; Li, Q.; Luo, L.; Zhang, M.; Yuan, L.-M. Homochiral metal-organic framework immobilized on silica gel by the interfacial polymerization for HPLC enantioseparations. J. Liq. Chromatogr. Relat. Technol. 2018, 1, 1-7. [CrossRef]

109. Lang, L.; Shengming, X.; Junhui, Z.; Ling, C.; Pengjing, Z.; Liming, Y. A Gas Chromatographic Stationary of Homochiral Metal-peptide Framework Material and Its Applications. Chem. Res. Chin. Univ. 2017, 33, $24-30$.

110. Zheng, D.-D.; Wang, L.; Yang, T.; Zhang, Y.; Wang, Q.; Kurmoo, M.; Zeng, M.-H. A Porous Metal-Organic Framework $\left[\mathrm{Zn}_{2}(\mathrm{bdc})(\mathrm{L}-\mathrm{lac})\right]$ as a Coating Material for Capillary Columns of Gas Chromatography. Inorg. Chem. 2017, 56, 11043-11049. [CrossRef] [PubMed]

111. Zheng, D.-D.; Zhang, Y.; Wang, L.; Kurmoo, M.; Zeng, M.-H. A rod-spacer mixed ligands MOF $\left[\mathrm{Mn}_{3}(\mathrm{HCOO})_{2}(\mathrm{Dcam})_{2}(\mathrm{DMF})_{2}\right]_{\mathrm{n}}$ as coating material for gas chromatography capillary column. Inorg. Chem. Com. 2017, 82, 34-38. [CrossRef]

112. Tang, P.; Wang, R.; Chen, Z. In situ growth of Zr-based metal-organic framework UiO-66- $\mathrm{NH}_{2}$ for open-tubular capillary electrochromatography. Electrophoresis 2018, 39, 2619-2625. [CrossRef] [PubMed]

113. Li, Z.; Mao, Z.; Chen, Z. In-situ growth of a metal organic framework composed of zinc(II), adeninate and biphenyldicarboxylate as a stationary phase for open-tubular capillary electrochromatography. Microchim. Acta 2019, 186, 53-61. [CrossRef]

114. Wang, X.; Ye, N.; Hu, X.; Liu, Q.; Li, J.; Peng, L.; Ma, X. Open-tubular capillary electrochromatographic determination of ten sulfonamides in tap water and milk by a metal-organic framework-coated capillary column. Electrophoresis 2018, 39, 2236-2245. [CrossRef]

115. Pan, C.; Lv, W.; Niu, X.; Wang, G.; Chen, H.; Chen, X. Homochiral zeolite-like metal-organic framework with DNA like double-helicity structure as stationary phase for capillary electrochromatography enantioseparation. J. Chromatogr. A 2018, 1541, 31-38. [CrossRef]

116. Tachikawa, T.; Choi, J.R.; Fujitsuka, M.; Majima, T. Photoinduced charge-transfer processes on MOF-5 nanoparticles: Elucidating differences between metal-organic frameworks and semiconductor metal oxides. J. Phys. Chem. C 2008, 112, 14090-14101. [CrossRef]

117. Ji, L.; Jin, Y.; Wu, K.; Wan, C.; Yang, N.; Tang, Y. Morphology-dependent electrochemical sensing performance of metal (Ni, Co, Zn)-organic frameworks. Anal. Chim. Acta 2018, 1031, 60-66. [CrossRef] [PubMed]

118. Hu, J.-S.; Dong, S.-J.; Wu, K.; Zhang, X.-L.; Jiang, J.; Yuan, J.; Zheng, M.-D. An ultrastable magnesium-organic framework as multi-responsive luminescent sensor for detecting trinitrotoluene and metal ions with high selectivity and sensitivity. Sens. Actuat. B 2019, 283, 255-261. [CrossRef] 
119. Xu, N.; Zhang, Q.; Hou, B.; Cheng, Q.; Zhang, G. A Novel Magnesium Metal-Organic Framework as a Multiresponsive Luminescent Sensor for Fe(III) Ions, Pesticides, and Antibiotics with High Selectivity and Sensitivity. Inorg. Chem. 2018, 57, 13330-13340. [CrossRef] [PubMed]

120. Zhang, F.; Yao, H.; Zhao, Y.; Li, X.; Zhang, G.; Yang, Y. Mixed matrix membranes incorporated with Ln-MOF for selective and sensitive detection of nitrofuran antibiotics based on inner filter effect. Talanta 2017, 174, 660-666. [CrossRef] [PubMed]

121. Asadi, F.; Azizi, S.N.; Ghasemi, S. Preparation of Ag nanoparticles on nano cobalt-based metal organic framework (ZIF-67) as catalyst support for electrochemical determination of hydrazine. J. Mater. Sci. Mater. Electron. 2019, 30, 5410-5420. [CrossRef]

122. Peng, M.; Guan, G.; Deng, H.; Han, B.; Tian, C.; Zhuang, J.; Xu, Y.; Liu, W.; Lin, Z. PCN-224/rGO nanocomposite based photoelectrochemical sensor with intrinsic recognition ability for efficient $\mathrm{p}$-arsanilic acid detection. Environ. Nano 2019, 6, 207-215. [CrossRef]

123. Zhou, Y.; Li, C.; Li, X.; Zhu, X.; Ye, B.; Xu, M. A sensitive aptasensor for the detection of b-amyloid oligomers based on metal-organic frameworks as electrochemical signal probes. Anal. Methods 2018, 10, 4430-4437. [CrossRef]

124. Sachdeva, S.; Koper, S.J.H.; Sabetghadam, A.; Soccol, D.; Gravesteijn, D.J.; Kapteijn, F.; Sudhölter, E.J.R.; Gascon, J.; de Smet, L.C.P.M. Gas Phase Sensing of Alcohols by Metal Organic Framework-Polymer Composite Materials. ACS Appl. Mater. Interf. 2017, 9, 24926-24935. [CrossRef]

125. Zhou, N.; Ma, Y.; Hu, B.; He, L.; Wang, S.; Zhang, Z.; Luc, S. Construction of Ce-MOF@COF hybrid nanostructure: Label-free aptasensor for the ultrasensitive detection of oxytetracycline residues in aqueous solution environments. Biosens. Bioelectron. 2019, 127, 92-100. [CrossRef]

126. Li, J.; Xia, J.; Zhang, F.; Wang, Z.; Liu, Q. An electrochemical sensor based on copper-based metal-organic frameworks-graphene composites for determination of dihydroxybenzene isomers in water. Talanta 2018, 181, 80-86. [CrossRef]

127. Das, A.; Biswas, S. A multi-responsive carbazole-functionalized $\mathrm{Zr}$ (IV)-based metal-organic framework for selective sensing of Fe(III), cyanide and p-nitrophenol. Sens. Actuat. B 2017, 250, 121-131. [CrossRef]

128. Guo, X.-Y.; Dong, Z.-P.; Zhao, F.; Liu, Z.-L.; Wang, Y.-Q. Zinc(II)-organic framework as a multi-responsive photoluminescence sensor for efficient and recyclable detection of pesticide 2,6-dichloro- 4-nitroaniline, Fe(III) and Cr(VI). New J. Chem. 2019, 43, 2353-2361. [CrossRef] 